\title{
Probabilistic sensitivity analysis of biochemical reaction systems
}

\author{
Hong-Xuan Zhang, ${ }^{1}$ William P. Dempsey, Jr., ${ }^{2}$ and John Goutsias ${ }^{1, a)}$ \\ ${ }^{1}$ The Whitaker Biomedical Engineering Institute, Johns Hopkins University, Baltimore, \\ Maryland 21218, USA \\ ${ }^{2}$ Division of Bioengineering, California Institute of Technology, Pasadena, California 91125, USA
}

(Received 22 May 2009; accepted 24 July 2009; published online 1 September 2009)

\begin{abstract}
Sensitivity analysis is an indispensable tool for studying the robustness and fragility properties of biochemical reaction systems as well as for designing optimal approaches for selective perturbation and intervention. Deterministic sensitivity analysis techniques, using derivatives of the system response, have been extensively used in the literature. However, these techniques suffer from several drawbacks, which must be carefully considered before using them in problems of systems biology. We develop here a probabilistic approach to sensitivity analysis of biochemical reaction systems. The proposed technique employs a biophysically derived model for parameter fluctuations and, by using a recently suggested variance-based approach to sensitivity analysis [Saltelli et al., Chem. Rev. (Washington, D.C.) 105, 2811 (2005)], it leads to a powerful sensitivity analysis methodology for biochemical reaction systems. The approach presented in this paper addresses many problems associated with derivative-based sensitivity analysis techniques. Most importantly, it produces thermodynamically consistent sensitivity analysis results, can easily accommodate appreciable parameter variations, and allows for systematic investigation of high-order interaction effects. By employing a computational model of the mitogen-activated protein kinase signaling cascade, we demonstrate that our approach is well suited for sensitivity analysis of biochemical reaction systems and can produce a wealth of information about the sensitivity properties of such systems. The price to be paid, however, is a substantial increase in computational complexity over derivative-based techniques, which must be effectively addressed in order to make the proposed approach to sensitivity analysis more practical. (C) 2009 American Institute of Physics.
\end{abstract}

[doi:10.1063/1.3205092]

\section{INTRODUCTION}

A fundamental problem in computational systems biology is the construction of biochemical reaction system models that can effectively predict cellular behavior. ${ }^{1,2}$ Subsequent analysis of such models may reveal a wealth of biologically relevant information, including a list of biochemical factors (e.g., biochemical reactions and molecular species) that are most influential in shaping cellular responses. Determining the most influential factors in a biochemical reaction system is an important problem with many applications. For example, identifying influential biochemical factors and targeting these factors with high specificity is a promising pharmacological intervention approach for treating human diseases. ${ }^{3}$

A powerful tool for studying the properties of a biochemical reaction system is sensitivity analysis. ${ }^{4,5}$ The objective of sensitivity analysis is to determine the biochemical factors that produce no noticeable variations in system response and identify those factors that are most influential in shaping that response. Sensitivity analysis has been applied in many diverse fields of science and engineering, including mechanical engineering, ${ }^{6,7}$ environmental engineering, ${ }^{8,9}$ pharmacology, ${ }^{3,10}$ biochemistry, ${ }^{5,11,12}$ and finance. ${ }^{13,14}$ In sys-

\footnotetext{
a) Author to whom correspondence should be addressed. Tel.: 410-516-7871. FAX: 410-516-4594. Electronic mail: goutsias@jhu.edu.
}

tems biology, sensitivity analysis has allowed researchers to identify factors controlling biological behavior in cells, ${ }^{15-18}$ simplify procedures for designing and optimizing genetic circuits, ${ }^{19}$ obtain insights into the robustness and fragility tradeoff in cell regulation, ${ }^{20}$ and determine appropriate targets for pharmacological intervention..$^{10,21}$

The sensitivity analysis approaches available in the literature can be classified into two groups. The first group deals primarily with deterministic techniques for sensitivity analysis, primarily based on derivatives of a response function of interest with respect to system parameters. The second group deals with probabilistic (stochastic) techniques, which quantify statistical fluctuations in system response due to random fluctuations in factors of interest.

Derivative-based sensitivity analysis techniques are subject to several drawbacks, which must be carefully considered before applying these techniques to problems of systems biology. For example, derivatives are most often used to evaluate the effects of infinitesimal changes in parameter values on the system response. This is usually done by ignoring important biophysical and thermodynamic properties, which may strongly constrain the biochemical reaction system at hand. ${ }^{22}$ Moreover, the derivatives are calculated at reference parameter values, which must be the true values for the results to be biologically relevant. However, the true parameter values of a real biochemical reaction system are rarely known. Instead, they are substituted by estimated val- 
ues, whose accuracy is often affected by unpredictable biological and experimental variability, which cannot be accounted for by a deterministic technique. The issue here is that different reference parameter values may produce different sensitivity results. As a consequence, the use of a derivative-based sensitivity analysis technique may easily lead to biologically false conclusions, since it cannot deal effectively with uncertainty.

Another important problem associated with derivativebased sensitivity analysis is that simple derivative calculations at a given set of reference values will most certainly fail to reveal important sensitivity properties due to substantial parameter variations. In certain cases, we may be able to address this problem by averaging derivative values calculated at appropriately selected points in the parameter space. ${ }^{11}$ It is expected, however, that for appreciable parameter variations, such averaging will not provide an accurate description of the sensitivity properties of a biochemical reaction system. As a matter of fact, it has been pointed out by Saltelli et $a .^{23}$ that although derivative averaging leads to a useful method for sensitivity analysis (known as elementary effect test), it should only be used to derive approximate sensitivity information, since it may introduce appreciable errors in the analysis.

In addition to the above, the task of accurately calculating response derivatives is not straightforward. One may easily express the response derivatives in terms of concentration sensitivities and analytically derive a system of differential equations that govern the dynamic evolution of these sensitivities. Then, evaluation of response derivatives will require simultaneous integration of the sensitivity equations together with the differential equations governing the dynamic evolution of the underlying molecular concentrations. Most often this step cannot be implemented in a reasonable time due to stiffness of the underlying differential equations. ${ }^{4}$ As a consequence, most users of derivative-based sensitivity analysis techniques resort to approximating derivatives by finite differences. However, the resulting approximations must be carefully used in applications, since it is difficult to theoretically predict, control, and numerically evaluate their accuracy. ${ }^{4,11}$

Finally, most derivative-based sensitivity analysis techniques employed in the literature use first-order derivatives to quantify the influence of a single parameter on the system response, while fixing the remaining parameters to their true values. This is not an appropriate strategy in most problems of systems biology, since control of biological behavior is usually exerted by the orchestrated influence of many biochemical factors. In our opinion, sensitivity analysis of a biochemical reaction system must be done by a method that considers the simultaneous influence of various biochemical factors on the system response. This is of particular interest in pharmacological applications of sensitivity analysis, since molecular-based drug design techniques must consider the biological effects of targeting several biochemical factors simultaneously.

Although sensitivities with respect to two or more factors can be well defined by means of second- and higherorder derivatives, accurate evaluation of such derivatives is a much more difficult problem than calculating first-order derivatives. Therefore, and in view of our previous discussion, we do not recommend use of second- and higher-order derivatives for sensitivity analysis of biochemical reaction systems. Most importantly, and due to their local nature, these derivatives cannot capture real joint sensitivity effects, which often occur at appreciable levels of parameter variations,

Due to the previous drawbacks, we believe that the general use of derivative-based sensitivity analysis techniques in systems biology must be limited, despite their extensive use in the literature. In this paper we demonstrate that probabilistic sensitivity analysis, and more precisely the variancebased sensitivity analysis approach developed by Sobol ${ }^{24,25}$ and Saltelli et al. ${ }^{23,26}$ is better suited for biochemical reaction system analysis. The work presented in this paper demonstrates that variance-based sensitivity analysis can easily address the previous drawbacks associated with derivativebased techniques. The price to be paid, however, is a substantial increase in computational complexity. This problem must be effectively addressed by future research in order to make variance-based sensitivity analysis more practical in problems of systems biology.

In this paper, we develop a variance-based sensitivity analysis technique that takes into account basic biophysical and thermodynamic properties underlying a biochemical reaction system. The proposed technique employs a biophysically derived model for parameter fluctuations, is less sensitive to the choice of reference values, can easily accommodate appreciable parameter variations, and allows for a systematic investigation of high-order interaction effects among system components.

In Sec. II, we provide a mathematical description for biochemical reaction systems modeled by classical chemical kinetics and identify appropriate system response functions for sensitivity analysis. By using basic thermodynamic principles, we develop in Sec. III a probabilistic model for the rate constants of a biochemical reaction system. We employ this model to identify appropriate biophysical factors of interest to sensitivity analysis and mathematically characterize their fluctuations. In Sec. IV, we review a variance-based sensitivity analysis technique and discuss its applicability to biochemical reaction systems. By limiting our interest to first- and second-order effects, we present a systematic methodology for classifying biochemical factors (reactions and molecular species) based on how these factors influence the system response. Although a substantial portion of this section constitutes a review of variance-based sensitivity analysis, we think that the material presented in this section is necessary, since the majority of investigators dealing with biological system analysis are not aware of this methodology. In Sec. V, we present a set of Monte Carlo estimators that we use to estimate the quantities required for implementing the proposed variance-based sensitivity analysis approach, whereas, in Sec. VI, we apply our method on a previously suggested model of the mitogen-activated protein kinase (MAPK) signaling cascade. We use the results obtained by variance-based sensitivity analysis to identify the reactions and molecular species that are most important for controlling appropriately chosen response characteristics of 
the MAPK cascade. Our analysis agrees well with published experimental conclusions and clearly demonstrates the potential of variance-based techniques for sensitivity analysis of biochemical reaction systems. Finally, we discuss several important issues in Sec. VII, whereas in Sec. VIII we present our conclusions.

\section{BIOCHEMICAL REACTION SYSTEMS}

In this paper, we consider a well-stirred (homogeneous) biochemical reaction system at constant temperature and volume that consists of $M$ coupled reversible reactions,

$$
\sum_{n=1}^{N} \nu_{n m} X_{n} \underset{k_{2 m}}{\stackrel{k_{2 m-1}}{\rightleftarrows}} \sum_{n=1}^{N} \nu_{n m}^{\prime} X_{n}, \quad m=1,2, \ldots, M,
$$

where $k_{2 m-1}, k_{2 m}$ are the rate constants of the forward and reverse reactions and $\nu_{n m}, \nu_{n m}^{\prime} \geq 0$ are the stoichiometry coefficients of the reactants and products. The system contains $N$ molecular species $X_{1}, X_{2}, \ldots, X_{N}$ whose concentrations at time $t \geq 0$ are denoted by $x_{1}(t), x_{2}(t), \ldots, x_{N}(t)$, respectively. If we assume that the molecular concentrations evolve continuously as a function of time and that all reactions are sufficiently characterized by the mass action rate law, then we can characterize the concentration dynamics by the following chemical kinetic equations: ${ }^{27}$

$$
\begin{aligned}
\frac{d x_{n}(t)}{d t}= & \sum_{m=1}^{M} s_{n m}\left\{k_{2 m-1} \prod_{i=1}^{N}\left[x_{i}(t)\right]^{\nu_{i m}}-k_{2 m} \prod_{i=1}^{N}\left[x_{i}(t)\right]^{\nu_{i m}^{\prime}}\right\}, \\
& t \geq 0, \quad n=1,2, \ldots, N,
\end{aligned}
$$

where

$$
s_{n m}:=\nu_{n m}^{\prime}-\nu_{n m}
$$

Our objective is to use sensitivity analysis to quantify the relative importance of each reaction or molecular species in influencing a response characteristic of the system.

To determine appropriate system responses, we focus our attention on the concentration profile $x_{n}(t)$ of a particular molecular species $n$ within an observation time interval $\left[0, t_{\max }\right]$ and consider three features of interest, namely, the timing $T_{n}$, duration $D_{n}$, and strength $S_{n}$ defined by

$$
\begin{aligned}
& T_{n}: \int_{0}^{T_{n}} x_{n}(t) d t=\int_{T_{n}}^{t_{0}} x_{n}(t) d t, \\
& D_{n}:=t_{0}, \\
& S_{n}:=\frac{1}{t_{0}} \int_{0}^{t_{0}} x_{n}(t) d t,
\end{aligned}
$$

where $t_{0}$ is the time at which the concentration becomes zero; see Fig. $1{ }^{28}$ Note that $T_{n}$ is the time at which the cumulative concentration is divided into two equal parts, whereas $S_{n}$ is the average concentration during the time interval $\left[0, t_{0}\right]$. The use of these quantities as response characteristics for sensitivity analysis of certain biological systems has been justified in the literature and strongly supported by experimental data. ${ }^{29}$ Note that a practical way to approximate the duration is to determine the value of $D_{n}$ such that

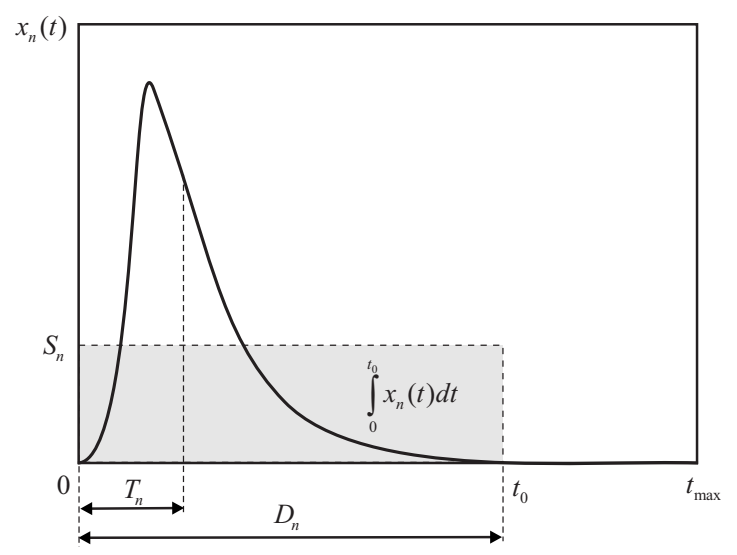

FIG. 1. The timing $T_{n}$, duration $D_{n}$, and strength $S_{n}$ of the concentration profile $x_{n}(t)$ of a molecular species $n$.

$$
\int_{0}^{D_{n}} x_{n}(t) d t=(1-\varepsilon) \int_{0}^{t_{\max }} x_{n}(t) d t
$$

for a sufficiently small positive number $\varepsilon$.

We will take the system response to be the logarithm of timing, duration, or strength. The reason for this choice is that typically, the probability density functions of these quantities are long tailed. Consequently, there is an appreciable probability for outliers, which may seriously compromise the numerical evaluation of response variances. Since we evaluate sensitivity indices in this paper by calculating conditional and unconditional response variances, we need to reduce the effect of outliers. We can achieve this objective by considering $\log$ responses. We conjecture that a biological system is not sensitive to the response value per se but to its log value. Naturally, this provides robustness with noticeable changes occurring only with strong and sustained variations in system response.

\section{PROBABILISTIC MODELING OF RATE CONSTANTS}

We can develop a powerful methodology for sensitivity analysis of biochemical reaction systems by recognizing that the rate constants of the underlying reactions may fluctuate randomly (e.g., due to unpredictable environmental, biological, and biochemical conditions) and by assessing how these fluctuations affect the system response. To achieve this, we consider the biochemical reaction system modeled by Eqs. (1) and (2). We will assume here that the system contains a large fraction of inert molecular species whose total concentration $x_{\text {tot }}$ does not vary with time. ${ }^{22}$ We can normalize Eq. (2) by dividing both sides with respect to $x_{\text {tot }}$, in which case,

$$
\begin{gathered}
\frac{d q_{n}(t)}{d t}=\sum_{m=1}^{M} s_{n m}\left\{\kappa_{2 m-1} \prod_{i=1}^{N}\left[q_{i}(t)\right]^{\nu_{i m}}-\kappa_{2 m} \prod_{i=1}^{N}\left[q_{i}(t)\right]^{\nu_{i m}^{\prime}}\right\}, \\
t \geq 0, \quad n=1,2, \ldots, N,
\end{gathered}
$$

where

$$
q_{n}(t):=\frac{x_{n}(t)}{x_{\mathrm{tot}}},
$$




$$
\begin{gathered}
\kappa_{2 m-1}:=\frac{k_{2 m-1}}{x_{\text {tot }}} \prod_{n=1}^{N} x_{\text {tot }}^{\nu_{n m}}, \\
\kappa_{2 m}:=\frac{k_{2 m}}{x_{\text {tot }}} \prod_{n=1}^{N} x_{\text {tot }}^{\nu_{n m}^{\prime}} .
\end{gathered}
$$

Note that $q_{n}$ is dimensionless, whereas $\kappa_{2 m-1}$ and $\kappa_{2 m}$ have units $\mathrm{s}^{-1}$. We refer to $\kappa_{2 m-1}$ and $\kappa_{2 m}$ as normalized rate constants.

We assume that the reactants and products form a homogeneous mixture of $N$ molecular species $X_{1}, X_{2}, \ldots, X_{N}$ in solution. Basic thermodynamic arguments imply the following expressions for the forward and reverse normalized rate constants:

$$
\begin{gathered}
\kappa_{2 m-1}=\frac{k_{B} T}{h} \frac{c_{m}^{\ddagger}}{\prod_{n=1}^{N} c_{n}^{\nu_{n m}}}, \\
\kappa_{2 m}=\frac{k_{B} T}{h} \frac{c_{m}^{\ddagger}}{\prod_{n=1}^{N} c_{n}^{\nu_{n m}^{\prime}}},
\end{gathered}
$$

which are known as Eyring-Polanyi equations. ${ }^{30}$ In these expressions, $k_{B}$ is the Boltzmann constant $\left(k_{B}=1.3806504\right.$ $\left.\times 10^{-23} \mathrm{~J} \mathrm{~K}^{-1}\right), T$ is the system temperature, $h$ is the Planck constant $\left(h=6.62606885 \times 10^{-34} \mathrm{~J} \mathrm{~s}\right), c_{m}^{\ddagger}$ is the capacity of the activated complex associated with the $m^{\text {th }}$ reaction, and $c_{n}$ is the capacity of the $n^{\text {th }}$ molecular species. The capacities are defined by

$$
\begin{aligned}
& c_{m}^{\ddagger}:=\exp \left\{-\frac{\mu_{m}^{\ddagger 0}}{k_{B} T}\right\}, \quad m=1,2, \ldots, M, \\
& c_{n}:=\exp \left\{-\frac{\mu_{n}^{0}}{k_{B} T}\right\}, \quad n=1,2, \ldots, N,
\end{aligned}
$$

where $\mu_{m}^{\ddagger 0}$ is the standard chemical potential of the activated complex associated with the $m^{\text {th }}$ reaction and $\mu_{n}^{0}$ is the standard chemical potential of the $n^{\text {th }}$ molecular species. ${ }^{31}$

Due to unpredictable biological variability, there is a great deal of uncertainty regarding the exact value of the standard chemical potential of a reaction. We may assume that the standard chemical potential of the activated complex associated with the $m^{\text {th }}$ reaction is a random variable $M_{m}^{\ddagger 0}$ given by ${ }^{32}$

$$
M_{m}^{\ddagger 0}=\mu_{m}^{\ddagger 0}+k_{B} T Y_{m}^{\ddagger}, \quad m=1,2, \ldots, M,
$$

where $\mu_{m}^{\ddagger 0}$ is a nominal value and $Y_{m}^{\ddagger}$ is a zero mean random variable that accounts for variations of the standard chemical potential about its nominal value. A possible source of variation for the standard chemical potential of the activated complex is unpredictable fluctuations in the conformational states and concentrations of enzymes responsible for catalyzing the reaction. ${ }^{33,34}$ It is well known that enzymes enhance the reaction rate by lowering the standard chemical potential of the activated complex. ${ }^{35}$ It is therefore expected that uncertainty in the conformational state and concentration of an enzyme catalyzing a given reaction will produce uncertainty in the value of the standard chemical potential of the activated complex of that reaction.

Similarly, we may assume that the standard chemical potential of the $n^{\text {th }}$ molecular species is a random variable $M_{n}^{0}$ given by

$$
M_{n}^{0}=\mu_{n}^{0}+k_{B} T Y_{n}, \quad n=1,2, \ldots, N,
$$

where $\mu_{n}^{0}$ is a nominal value and $Y_{n}$ is a zero mean random variable that accounts for variations in the standard chemical potential about its nominal value. A possible source of variation in the standard chemical potential of a molecular species, such as a protein, is genetic point (single nucleotide) mutations, which may occur randomly and may result in a small change in the amino acid sequence of the protein. Because of redundancy of the genetic code (64 codons and only 20 amino acids) and the relative closeness within the code of biochemically similar amino acids, most small changes in proteins will not affect the stoichiometry of the underlying biochemical reaction network but may alter the kinetic properties of the participating molecular species by modifying the standard chemical potential (or capacity) values.

As a consequence of our previous discussion, we can now treat the normalized rate constants as random variables $K_{2 m-1}$ and $K_{2 m}$ given by

$$
\begin{gathered}
K_{2 m-1}=\frac{k_{B} T}{h} \frac{C_{m}^{\ddagger}}{\prod_{n=1}^{N} C_{n}^{\nu_{n m}}}, \\
K_{2 m}=\frac{k_{B} T}{h} \frac{C_{m}^{\ddagger}}{\prod_{n=1}^{N} C_{n}^{\nu_{n m}^{\prime}}},
\end{gathered}
$$

where the (random) capacities $C_{m}^{\ddagger}$ and $C_{n}$ are defined by

$$
\begin{aligned}
& C_{m}^{\ddagger}:=\exp \left\{-\frac{M_{m}^{\ddagger}}{k_{B} T}\right\}, \quad m=1,2, \ldots, M, \\
& C_{n}:=\exp \left\{-\frac{M_{n}^{0}}{k_{B} T}\right\}, \quad n=1,2, \ldots, N .
\end{aligned}
$$

From Eqs. (8)-(13), we have that

$$
\begin{aligned}
& K_{2 m-1}=\kappa_{2 m-1} \exp \left\{-Y_{m}^{\ddagger}\right\} \exp \left\{\sum_{n=1}^{N} \nu_{n m} Y_{n}\right\}, \\
& K_{2 m}=\kappa_{2 m} \exp \left\{-Y_{m}^{\ddagger}\right\} \exp \left\{\sum_{n=1}^{N} \nu_{n m}^{\prime} Y_{n}\right\},
\end{aligned}
$$

where $\kappa_{2 m-1}$ and $\kappa_{2 m}$ are given by Eqs. (8) and (9). ${ }^{36}$

For biochemical reaction systems that do not contain spontaneous reactions (which are the types of systems we are interested in this paper), the standard chemical potential of the activated complex associated with a reaction must always be larger than the total standard chemical potential of the reactants or products of the reaction. This implies that we must satisfy the following inequalities: 


$$
M_{m}^{\ddagger 0} \geq \max \left\{\sum_{n=1}^{N} \nu_{n m} M_{n}^{0}, \sum_{n=1}^{N} \nu_{n m}^{\prime} M_{n}^{0}\right\}, \quad m=1,2, \ldots, M .
$$

In turn, we must have that

$$
\begin{aligned}
\mu_{m}^{\ddagger 0}+k_{B} T Y_{m}^{\ddagger} \geq & \max \left\{\sum_{n=1}^{N} \nu_{n m} \mu_{n}^{0}+k_{B} T \sum_{n=1}^{N} \nu_{n m} Y_{n},\right. \\
& \left.\sum_{n=1}^{N} \nu_{n m}^{\prime} \mu_{n}^{0}+k_{B} T \sum_{n=1}^{N} \nu_{n m}^{\prime} Y_{n}\right\}
\end{aligned}
$$

by virtue of Eqs. (10) and (11), or

$$
\begin{aligned}
& -Y_{m}^{\ddagger}+\sum_{n=1}^{N} \nu_{n m} Y_{n} \leq \ln \frac{k_{B} T}{h \kappa_{2 m-1}} \text { and } \\
& -Y_{m}^{\ddagger}+\sum_{n=1}^{N} \nu_{n m}^{\prime} Y_{n} \leq \ln \frac{k_{B} T}{h \kappa_{2 m}}
\end{aligned}
$$

by virtue of Eqs. (8) and (9). As a consequence, given biologically reasonable nominal values for $\kappa_{2 m-1}$ and $\kappa_{2 m}$, we need to make sure that the random variables $Y_{m}^{\ddagger}$ and $Y_{n}$ satisfy the inequalities imposed by Eq. (15). We will return to this issue in Sec. V.

In the following, we assume that $Y_{m}^{\ddagger}$ is a zero-mean Gaussian random variable with standard deviation $\lambda_{m}^{\ddagger}$, i.e., $Y_{m}^{\ddagger} \sim \mathcal{N}\left(0, \lambda_{m}^{\ddagger}\right)$ for $m=1,2, \ldots, M$. Then, Eq. (10) implies that the standard chemical potential $M_{m}^{\sharp 0}$ of the activated complex of the $m^{\text {th }}$ reaction is a Gaussian random variable with mean value $\mu_{m}^{\ddagger 0}$ and standard deviation $k_{B} T \lambda_{m}^{\ddagger}$. Similarly, we assume that $Y_{n}$ is a zero-mean Gaussian random variable with standard deviation $\lambda_{n}$; i.e., $Y_{n} \sim \mathcal{N}\left(0, \lambda_{n}\right)$ for $n=1,2, \ldots, N$. In this case, Eq. (11) implies that the standard chemical potential $M_{n}^{0}$ of the $n^{\text {th }}$ species is a Gaussian random variable with mean value $\mu_{n}^{0}$ and standard deviation $k_{B} T \lambda_{n}$. Finally, we assume that the random variables $\left\{Y_{m}^{\ddagger}, m=1,2, \ldots, M\right\}$ and $\left\{Y_{n}, n=1,2, \ldots, N\right\}$ are mutually independent.

The use of Gaussian distributions for modeling $Y_{m}^{\ddagger}$ and $Y_{n}$ cannot be easily justified experimentally. However, it is a convenient choice that can be viewed as an effective approximation of the actual distributions of $Y_{m}^{\ddagger}$ and $Y_{n}$, obtained by setting all higher-order $(\geq 3)$ statistical moments equal to zero. As a consequence, the probability distributions of the forward and reverse reaction rates are log normal. Interestingly, it has been argued in the literature that lognormal distributions are natural choices for modeling processes that evolve by energy transduction mechanisms that decrease free energy, ${ }^{37}$ which is the case in biochemical reaction systems.

The assumption of statistical independence between the random variables $\left\{Y_{m}^{\ddagger}, m=1,2, \ldots, M\right\}$ and $\left\{Y_{n}, n=1,2\right.$, $\ldots, N\}$ can be justified by arguing that the primary sources of variation in the standard chemical potentials of activated complexes and molecular species are different (e.g., fluctuations in enzyme concentrations versus genetic point mutations) and do not influence each other. However, it is more difficult to justify mutual independence within $\left\{Y_{m}^{\ddagger}, m\right.$
$=1,2, \ldots, M\}$ and $\left\{Y_{n}, n=1,2, \ldots, N\right\}$. We simply view this as a convenient approximating assumption that allows us to proceed with the sensitivity analysis approach we discuss in Sec. IV.

Equation (14) suggests that variations in the forward and reverse reaction rates may occur due to variations in the standard chemical potential of the activated complex associated with that reaction and variations in the standard chemical potentials of its reactants. These variations are modeled by two Gaussian random variables

$$
\begin{gathered}
G_{m}:=-Y_{m}^{\ddagger}+\sum_{n=1}^{N} \nu_{n m} Y_{n}, \\
G_{m}^{\prime}:=-Y_{m}^{\ddagger}+\sum_{n=1}^{N} \nu_{n m}^{\prime} Y_{n}
\end{gathered}
$$

for the forward and reverse reactions, respectively. Note that

$$
\begin{aligned}
& E\left[G_{m}\right]=0, \quad \operatorname{Var}\left[G_{m}\right]=\left[\lambda_{m}^{\ddagger}\right]^{2}+\sum_{n=1}^{N} \nu_{n m}^{2} \lambda_{n}^{2}, \\
& E\left[G_{m}^{\prime}\right]=0, \quad \operatorname{Var}\left[G_{m}^{\prime}\right]=\left[\lambda_{m}^{\ddagger}\right]^{2}+\sum_{n=1}^{N}\left[\nu_{n m}^{\prime}\right]^{2} \lambda_{n}^{2} .
\end{aligned}
$$

Therefore, and due to the dependence of $\operatorname{Var}\left[G_{m}\right]$ and $\operatorname{Var}\left[G_{m}^{\prime}\right]$ on the stoichiometry coefficients $\nu_{n m}$ and $\nu_{n m}^{\prime}$, the size of variations in the reaction rates will not be uniform in general, even if all standard deviations $\lambda_{m}^{\ddagger}$ and $\lambda_{n}$ take the same values, which is the case in Sec. VI. Note also that the probability distributions of $K_{2 m-1}$ and $K_{2 m}$ are log normal with median $\left[K_{2 m-1}\right]=\kappa_{2 m-1}$ and median $\left[K_{2 m}\right]=\kappa_{2 m}$.

To use Eq. (14) for sensitivity analysis, we must know the values of $\kappa_{2 m-1}$ and $\kappa_{2 m}$. Our model dictates that $\kappa_{2 m-1}$ and $\kappa_{2 m}$ must be the median values of the forward and reverse normalized rate constants given by Eq. (7). In many applications of interest, we obtain the values of the reaction rate constants $k_{2 m-1}$ and $k_{2 m}$ from the literature. However, to determine the normalized values $\kappa_{2 m-1}$ and $\kappa_{2 m}$, we must know the total concentration $x_{\text {tot }}$ of the inert species in the system, a quantity that is extremely difficult to measure. Note that if the response $R_{x}(\boldsymbol{k})$ of the biochemical system described by Eq. (2) satisfies

$$
R_{x}(\boldsymbol{k})=R_{q}(\boldsymbol{\kappa})+\gamma
$$

for some constant $\gamma$, where $R_{q}(\boldsymbol{\kappa})$ is the response of the system described by Eq. (6), then the variance-based sensitivity indices we derive from Sec. IV will be the same in both cases. Since $q_{n}(t)=x_{n}(t) / x_{\text {tot }}$, for every $t \geq 0$, the timing and duration satisfy Eq. (18) with $\gamma=0$, whereas the strength satisfies Eq. (18) with $\gamma=\ln x_{\text {tot. }}$ Therefore, the actual value of $x_{\text {tot }}$ is immaterial in our variance-based sensitivity analysis approach. As a result, we can set $x_{\text {tot }}=1$ and take the numerical values of $\kappa_{2 m-1}$ and $\kappa_{2 m}$ to be the published values of $k_{2 m-1}$ and $k_{2 m}$, respectively. However, we must make sure that these values lead to a thermodynamically feasible biochemical reaction system, in the sense that there exist capaci- 
ties $c_{m}^{\ddagger}, m=1,2, \ldots, M$, and $c_{n}, n=1,2, \ldots, N$, such that the Eyring-Polanyi equations are satisfied.

It has been recently pointed out in the literature that a serious issue associated with existing sensitivity analysis techniques is lack of thermodynamic consistency. ${ }^{22}$ However, an important consequence of Eqs. (8) and (14) is that

$$
\begin{aligned}
& \frac{\kappa_{2 m-1}}{\kappa_{2 m}}=\prod_{n=1}^{N} c_{n}^{s_{n m}} \quad \text { and } \\
& \frac{K_{2 m-1}}{K_{2 m}}=\frac{\kappa_{2 m-1}}{\kappa_{2 m}} \exp \left\{-\sum_{n=1}^{N} s_{n m} Y_{n}\right\}
\end{aligned}
$$

by virtue of Eq. (3), which are constraints thermodynamically imposed on the reaction rate constants. Note that if $\boldsymbol{b}$ is an $M \times 1$ vector in the null space of the $N \times M$ stoichiometry matrix $S=\left[s_{n m}\right]$ of the biochemical reaction system (i.e., if $\mathrm{S} \boldsymbol{b}=0$ ), then Eq. (19) implies that

$$
\prod_{m=1}^{M}\left(\frac{K_{2 m-1}}{K_{2 m}}\right)^{b_{m}}=\prod_{m=1}^{M}\left(\frac{\kappa_{2 m-1}}{\kappa_{2 m}}\right)^{b_{m}}=1,
$$

which are known as Wegscheider conditions. ${ }^{27}$ Equation (20) shows that the proposed probabilistic model for the reaction rate constants automatically satisfies the Wegscheider conditions, provided that these conditions are satisfied by the nominal (median) reaction rate constants $\kappa_{m}$. As a consequence, and in sharp contrast to existing techniques, the sensitivity analysis approach we present in this paper can produce thermodynamically consistent results.

Finally, we should note that some reactions in Eq. (1) may be "incomplete" in the sense that they are expressed without indicating every molecular species participating in the reaction, such as an enzyme catalyzing a particular reaction. For example, it is common to model phosphorylation of a protein $\mathrm{A}$ by a kinase $\mathrm{K}$ using the following reaction:

$$
\mathrm{A}+\mathrm{K} \stackrel{k}{\rightarrow} \mathrm{A}-\mathrm{P}+\mathrm{K} \text {. }
$$

However, phosphorylation must also include the cleavage of adenosine triphosphate (ATP) into adenosine diphosphate (ADP) and inorganic phosphate (P), which leads to the following more accurate reaction:

$$
\mathrm{A}+\mathrm{K}+\mathrm{ATP} \stackrel{k^{\prime}}{\rightarrow} \mathrm{A}-\mathrm{P}+\mathrm{K}+\mathrm{ADP} .
$$

Note, however, that if we set $k=k^{\prime} x_{\mathrm{ATP}}$, then the two reactions will be equivalent. Therefore, and in order to deal with an incomplete reaction, we will assume that its reaction rate is the actual rate multiplied by the corresponding concentrations of the "missing" reactants. In this case, fluctuations in the rate constant values may also be attributed to fluctuations in the concentrations of these reactants.

\section{VARIANCE-BASED SENSITIVITY ANALYSIS}

In Sec. III, we introduced a probabilistic model for the reaction rate constants of a biochemical reaction system by modeling random fluctuations in the standard chemical potentials of the activated complexes associated with the reac- tions and in the standard chemical potentials of the underlying molecular species. By assessing how these fluctuations affect the system response, we can classify reactions (molecular species) into two groups, namely, influential reactions (molecular species) and noninfluential reactions (molecular species). We say that a reaction (molecular species) is influential if random fluctuations in the corresponding standard chemical potential result in noticeable fluctuations in the system response. Otherwise, the reaction (molecular species) is said to be noninfluential.

In this section, we discuss a powerful approach to sensitivity analysis known as variance-based sensitivity analysis. $^{5,23,26}$ To simplify notation, we will use $U_{1}, U_{2}, \ldots, U_{J}$ to denote the random variables $Y^{\ddagger}$ and $Y$ associated with the standard chemical potentials. In general, we take $J=M+N$ and set $U_{j}=Y_{j}^{\ddagger}$ for $j=1,2, \ldots, M$ and $U_{M+j}=Y_{j}$ for $j=1,2, \ldots, N$. However, when the standard chemical potentials of the molecular species are fixed, we take $J=M$ and set $U_{j}=Y_{j}^{\ddagger}$ for $j=1,2, \ldots, M$, whereas when the standard chemical potentials of the activated complexes are fixed, we take $J=N$ and set $U_{j}=Y_{j}$ for $j=1,2, \ldots, N$. We will be referring to $U_{1}, U_{2}, \ldots, U_{J}$ as "biochemical factors."

Given a system response function $R(\boldsymbol{u})$, we can easily verify that its variance satisfies the following equation:

$$
V=\sum_{j=1}^{J} V_{j}+\sum_{j=1}^{J} \sum_{j^{\prime}>j} V_{j j^{\prime}}+\cdots+V_{12 \cdots J},
$$

where

$$
\begin{aligned}
V:= & \operatorname{Var}[R(\boldsymbol{U})] \\
V_{j}:= & \operatorname{Var}\left[E\left[R(\boldsymbol{U}) \mid U_{j}\right]\right], \\
V_{j j^{\prime}}:= & \operatorname{Var}\left[E\left[R(\boldsymbol{U}) \mid U_{j}, U_{j^{\prime}}\right]\right]-\operatorname{Var}\left[E\left[R(\boldsymbol{U}) \mid U_{j}\right]\right] \\
& -\operatorname{Var}\left[E\left[R(\boldsymbol{U}) \mid U_{j^{\prime}}\right]\right],
\end{aligned}
$$

with similar expressions for the remaining terms. If the biochemical factors $U_{1}, U_{2}, \ldots, U_{J}$ are statistically independent (which we have assumed here to be true), then each term on the right-hand side of Eq. (21) will be nonnegative (the $V_{j}$ terms are always non-negative). This result was first shown by Sobol ${ }^{24,25}$ and serves as the basis for constructing the sensitivity indices we discuss below.

Due to the well-known variance decomposition formula

$$
\operatorname{Var}[Y]=\operatorname{Var}[E[Y \mid X]]+E[\operatorname{Var}[Y \mid X]],
$$

Eq. (22) implies that

$$
V_{j}=\operatorname{Var}[R(\boldsymbol{U})]-E\left[\operatorname{Var}\left[R(\boldsymbol{U}) \mid U_{j}\right]\right] .
$$

As a consequence, $V_{j}$ quantifies the average reduction in response variance obtained by keeping the $j^{\text {th }}$ biochemical factor fixed. Therefore, we may use $V_{j}$ as a measure of the singular contribution of the $j^{\text {th }}$ biochemical factor to the system response. Likewise, from Eqs. (22)-(24), we obtain

$$
V_{j}+V_{j^{\prime}}+V_{j j^{\prime}}=\operatorname{Var}[R(\boldsymbol{U})]-E\left[\operatorname{Var}\left[R(\boldsymbol{U}) \mid U_{j}, U_{j^{\prime}}\right]\right] .
$$

Clearly, the term $V_{j j^{\prime}}$ quantifies the average reduction in the response variance due to jointly fixing the two biochemical 
factors $U_{j}$ and $U_{j^{\prime}}$, which is not accounted for by summing the average reductions obtained by separately fixing these factors. Hence, we may use $V_{j j^{\prime}}$ as a measure of the joint contribution of the biochemical factors $U_{j}$ and $U_{j^{\prime}}$ to the system response. Similar remarks apply for the higher-order terms on the right-hand side of Eq. (21). Clearly, Eq. (21) decomposes the response variance $V$ of a biochemical reaction system into a sum of individual terms, where each term quantifies the singular or joint contribution of a particular biochemical factor to the system response.

If the response function $R(\boldsymbol{u})$ is sufficiently smooth around $\mathbf{0}$, so that its derivatives of order $\geq 3$ at $\mathbf{0}$ are negligible, and if the biochemical factors $U_{j}$ are statistically independent zero-mean Gaussian random variables with sufficiently small standard deviations $\lambda_{j}$, so that

$$
\lambda_{j}^{2} \lambda_{j^{\prime}}^{2}\left[\frac{\partial^{2} R(\mathbf{0})}{\partial u_{j} \partial u_{j^{\prime}}}\right]^{2} \simeq 0, \quad \text { for every } j, j^{\prime}=1,2, \ldots, J,
$$

then $^{29}$

$$
V \simeq V_{1}+V_{2}+\cdots+V_{J}
$$

where

$$
V_{j} \simeq \lambda_{j}^{2}\left[\frac{\partial R(\mathbf{0})}{\partial u_{j}}\right]^{2}
$$

See also the discussion in Ref. 38. In this case, we can approximately decompose the response variance into a sum of individual terms $V_{j}, j=1,2, \ldots, J$. Then, the biochemical factors $U_{j}$ will mostly contribute to the response variance singularly. Note that if $R(\boldsymbol{u})$ is linear, then Eqs. (27) and (28) will be exact, regardless of the statistical model assumed for the biochemical factors $U_{j}$. More generally, Eq. (27) [but not necessarily Eq. (28)] is exact when the response function is additive. ${ }^{39}$ In both cases, Eq. (21) implies that all joint contributions of the biochemical factors $U_{1}, U_{2}, \ldots, U_{J}$ to the response variance will be zero.

In general, the total contribution of the $j^{\text {th }}$ biochemical factor to the response variance is given by

$$
C_{j}:=V_{j}+\sum_{j^{\prime} \neq j}^{J} V_{j j^{\prime}}+\cdots+V_{12 \cdots J},
$$

which implies that $V \leq \sum_{j=1}^{J} C_{j}$. Therefore, we cannot in general decompose the response variance into a sum of individual contributions from each biochemical factor.

From Eq. (29), we have that

$$
\tau_{j}:=\frac{C_{j}}{V}=\frac{V_{j}+\sum_{j^{\prime} \neq j}^{J} V_{j j^{\prime}}+\cdots+V_{12 \cdots J}}{V} .
$$

This index has been introduced as a tool for sensitivity analysis by Saltelli and his co-workers. ${ }^{5,23,26,40,41}$ It quantifies the fractional total (singular and joint) contribution of the $j^{\text {th }}$ biochemical factor to the response variance. For this reason, we refer to $\tau_{j}$ as the total-effect sensitivity index (TESI) of the $j^{\text {th }}$ biochemical factor. Note that

$$
0 \leq \tau_{j} \leq 1, \quad \text { for every } j=1,2, \ldots, J
$$

due to Eqs. (21) and (30). Moreover, it can be shown that

$$
\tau_{j}=\frac{E\left[\operatorname{Var}\left[R(\boldsymbol{U}) \mid \boldsymbol{U}_{(j)}\right]\right]}{\operatorname{Var}[R(\boldsymbol{U})]}=1-\frac{\operatorname{Var}\left[E\left[R(\boldsymbol{U}) \mid \boldsymbol{U}_{(j)}\right]\right]}{\operatorname{Var}[R(\boldsymbol{U})]},
$$

where $\boldsymbol{U}_{(j)}$ denotes the collection of all biochemical factors excluding $U_{j}{ }^{23}$ Therefore, $\tau_{j}$ is the average fractional response variance obtained when all factors, except the $j^{\text {th }}$ biochemical factor, are kept fixed.

An important objective of sensitivity analysis is to identify noninfluential biochemical factors (i.e., factors that do not appreciably influence the system response). This is of fundamental theoretical and experimental interest, since the biochemical reaction system will be robust to changes in the values of noninfluential factors, which can be fixed without appreciably affecting the system response. As a consequence, we may be able to ignore noninfluential biochemical factors, thus simplifying the complexity of the biochemical system under consideration. It is intuitive to believe that if a biochemical factor is noninfluential, fluctuations in its value will not appreciably affect the system response. This observation motivates us to define a biochemical factor as being noninfluential if stochastic fluctuations in its value does not generate noticeable fluctuations of the system response when the remaining factors are kept fixed. Based on this definition and Eq. (32), the $j^{\text {th }}$ biochemical factor is noninfluential if and only if $\tau_{j}=0$. Therefore, we can identify noninfluential biochemical factors as those with TESI values close to zero.

After sorting out the noninfluential biochemical factors, we would like to derive an index that we can use to rank the remaining factors based on how much these factors influence the system response. If we assume that Eq. (27) is satisfied, it will be natural to rank influential biochemical factors based on their corresponding $V_{j}$ values, since $V_{j}$ quantifies the contribution of the $j^{\text {th }}$ biochemical factor to the response variance. This leads to ranking influential biochemical factors based on the following index:

$$
\sigma_{j}:=\frac{V_{j}}{V}=\frac{\operatorname{Var}\left[E\left[R(\boldsymbol{U}) \mid U_{j}\right]\right]}{\operatorname{Var}[R(\boldsymbol{U})]} .
$$

It is clear from this formula that $\sigma_{j}$ quantifies the fractional singular contribution of the $j^{\text {th }}$ biochemical factor to the response variance. Therefore, we will refer to this quantity as the single-effect sensitivity index (SESI) of the $j^{\text {th }}$ biochemical factor. This index has been originally introduced by Iman, ${ }^{42}$ used by Krewski et al., ${ }^{10}$ and then by Saltelli et $a l .{ }^{5,23,26,41}$ Note that $0 \leq \sigma_{j} \leq 1$ for every $j=1,2, \ldots, J$ due to Eqs. (21) and (33). Thus, similar to the TESIs, the SESIs are also normalized to take values between 0 and 1 .

Subject to the assumptions that lead to Eqs. (27) and (28), we have that

$$
\tau_{j} \simeq \sigma_{j} \simeq \frac{\lambda_{j}^{2}\left[\partial R(\mathbf{0}) / \partial u_{j}\right]^{2}}{\sum_{j^{\prime}=1}^{J} \lambda_{j^{\prime}}^{2}\left[\partial R(\mathbf{0}) / \partial u_{j^{\prime}}\right]^{2}},
$$

which provides a direct link between derivative-based and variance-based sensitivity analysis techniques and shows that derivative-based sensitivity analysis may be viewed as a spe- 
TABLE I. Rules for interpreting the variance-based sensitivity indices.

\begin{tabular}{lll}
\hline \hline \multicolumn{1}{c}{$\sigma_{j} \simeq 0$} & \multicolumn{1}{c}{$\sigma_{j} \neq 0$} \\
\hline$\eta_{j} \simeq 0$ & $\begin{array}{l}\text { Factor } j \text { does not appreciably } \\
\text { influence the system response. }\end{array}$ & $\begin{array}{l}\text { Factor } j \text { influences the system } \\
\text { response mostly singularly. }\end{array}$ \\
$\eta_{j} \neq 0$ & $\begin{array}{l}\text { Factor } j \text { influences the system } \\
\text { response mostly jointly. }\end{array}$ & $\begin{array}{l}\text { Factor } j \text { influences the system } \\
\text { response both singularly and jointly. }\end{array}$ \\
\hline \hline
\end{tabular}

cial and restrictive case of variance-based sensitivity analysis. The form of SESI given by Eq. (34) has been proposed as a tool for sensitivity analysis by several investigators. ${ }^{5,23,26,38}$ However, its use requires verification of the assumptions associated with Eqs. (27) and (28), which is clearly very difficult to do in practice.

From Eqs. (21), (30), and (33), note that $\sigma_{j} \leq \tau_{j}$ for every $j=1,2, \ldots, J$, whereas

$$
\sum_{j=1}^{J} \sigma_{j} \leq 1 \leq \sum_{j=1}^{J} \tau_{j}
$$

The difference

$$
\delta:=1-\sum_{j=1}^{J} \sigma_{j}
$$

satisfies $0 \leq \delta \leq 1$. Moreover,

$$
\delta=\frac{1}{V}\left(V-\sum_{j=1}^{J} V_{j}\right)=\frac{1}{V}\left(\sum_{j=1}^{J} \sum_{j^{\prime}>j}^{J} V_{j j^{\prime}}+\cdots+V_{12 \cdots J}\right) .
$$

This shows that $\delta$ quantifies the fractional joint contribution of all biochemical factors to the response variance. Note that if the response function is additive, then $\delta=0$. If $\delta \simeq 0$, all joint contributions to the response variance will be negligible, whereas appreciable values of $\delta$ indicate that these contributions may be significant. In the former case, Eq. (27) will be approximately satisfied and we can thus justify ranking the influential reactions by using the SESIs.

When $\delta \neq 0,{ }^{43}$ we need to investigate whether a biochemical factor contributes to the system response singularly, jointly, or both. To do so, we may calculate the difference

$$
\eta_{j}:=\tau_{j}-\sigma_{j},
$$

which, together with Eqs. (30) and (33), leads to

$$
\eta_{j}=\frac{1}{V}\left(\sum_{j^{\prime} \neq j}^{J} V_{j j^{\prime}}+\cdots+V_{12 \cdots J}\right) .
$$

Equations (21) and (38) imply that $0 \leq \eta_{j} \leq 1$ for every $j=1,2, \ldots, J$. According to Eq. (38), $\eta_{j}$ quantifies the fractional contribution of the $j^{\text {th }}$ biochemical factor to the response variance jointly with one or more other factors. For this reason, we refer to $\eta_{j}$ as the joint-effect sensitivity index (JESI). If $\eta_{j} \simeq 0$, these contributions will be negligible, whereas appreciable values of $\eta_{j}$ indicate significant joint contributions. In the former case, we have $\tau_{j} \simeq \sigma_{j}$ by virtue of Eq. (37), which also implies that $\delta \simeq 0$ based on Eqs. (35) and (36). In this case, if $\sigma_{j} \simeq 0$, then $\tau_{j} \simeq 0$, and we may conclude that the $j^{\text {th }}$ biochemical factor does not appreciably influence the system response (i.e., it is noninfluential); whereas if $\sigma_{j} \neq 0$, then $\tau_{j} \neq 0$, and we may conclude that the $j^{\text {th }}$ biochemical factor influences the system response but mostly singularly. On the other hand, when $\eta_{j} \neq 0$, the singular and joint contribution of the $j^{\text {th }}$ biochemical factor to the response variance may be appreciable. In this case, if $\sigma_{j} \simeq 0$, we may conclude that the system response is not appreciably influenced by the $j^{\text {th }}$ factor alone, but mostly by the $j^{\text {th }}$ factor jointly with one or more other factors; whereas if $\sigma_{j} \neq 0$, we may conclude that the $j^{\text {th }}$ biochemical factor influences the response function both singularly and jointly with one or more other reactions. We summarize these remarks in Table I, which shows that the JESIs and SESIs are sufficient for proper classification of biochemical factors.

As we mentioned before, when the system response is not appreciably influenced by joint effects (i.e., when $\eta_{j} \simeq 0$ for $\left.j=1,2, \ldots, J\right)$, Eq. (27) is satisfied and we can use the SESIs (or the TESIs, since $\tau_{j} \simeq \sigma_{j}$ in this case) to rank the influential biochemical factors. When this is not true, we must identify an appropriate sensitivity index that we can use to rank the influential biochemical factors in the presence of joint effects. To do so, we first need to determine the purpose of identifying a biochemical factor as "more influential" than another factor and mathematically formalize what we mean by this comparison.

It is intuitive to believe that to maintain a robust behavior, a biochemical reaction system must control the standard chemical potentials associated with influential reactions and molecular species in a precise manner in order to reduce fluctuations in its response. This observation motivates us to define a biochemical factor as being the most influential if, by fixing its value, the system response variance is, on the average, the smallest possible. Likewise, we may define the second most influential biochemical factor, and so on. Now, if we go back to Eq. (24), we have that $E\left[\operatorname{Var}\left[R(\boldsymbol{U}) \mid U_{j}\right]\right]$ $=V-V_{j}$. This implies that on the average, the smallest response variance is obtained by fixing the biochemical factor with the largest $V_{j}$ value. As a consequence, we can still use the SESIs to rank the influential biochemical factors, even factors that jointly contribute to the response variance. This ranking can be useful even in cases of appreciable joint effects, especially when the problem is to influence the response of a biochemical reaction system by only targeting biochemical factors one at a time. However, we must always keep in mind that a ranking based on SESI values considers only the singular contribution of each biochemical factor to the response variance and does not take into account joint effects.

When $\eta_{j} \neq 0$, we may want to further investigate the joint 
influence of two biochemical factors $j$ and $j^{\prime}$ on the system response. We can achieve this objective by employing the following index:

$$
v_{j j^{\prime}}:=\frac{V_{j j^{\prime}}}{V}, \quad j^{\prime} \neq j,
$$

which we refer to as the pairwise-effect sensitivity index (PESI). From Eq. (31) and Eqs. (37)-(39), we have that $0 \leq v_{j j^{\prime}} \leq \eta_{j} \leq \tau_{j} \leq 1$ for $j^{\prime} \neq j$. If $v_{j j^{\prime}} \simeq 0$, we may conclude that the joint contribution of the biochemical factors $j$ and $j^{\prime}$ to the system response is negligible, whereas larger values of $v_{j j^{\prime}}$ indicate stronger joint contributions.

In addition to ranking individual biochemical factors, it may also be desirable to rank pairs of factors based on their contribution to the response variance. From Eq. (25), we have that $E\left[\operatorname{Var}\left[R(\boldsymbol{U}) \mid U_{j}, U_{j^{\prime}}\right]\right]=V-\left(V_{j}+V_{j^{\prime}}+V_{j j^{\prime}}\right)$. This implies that on the average, by fixing two factors, the smallest response variance is obtained when fixing the factors with the largest $V_{j}+V_{j^{\prime}}+V_{j j^{\prime}}$ value. As a consequence, we can evaluate the influence of pairs of biochemical factors on the system response by employing the following index:

$$
\sigma_{j j^{\prime}}:=\frac{V_{j}+V_{j^{\prime}}+V_{j j^{\prime}}}{V}=\frac{\operatorname{Var}\left[E\left[R(\boldsymbol{U}) \mid U_{j}, U_{j^{\prime}}\right]\right]}{\operatorname{Var}[R(\boldsymbol{U})]}, \quad j^{\prime} \neq j .
$$

We refer to $\sigma_{j j^{\prime}}$ as the double-effect sensitivity index (DESI). This index quantifies the average fractional reduction in the response variance when the two factors $j$ and $j^{\prime}$ are fixed. From Eqs. (21) and (40), we have that $0 \leq \sigma_{j j^{\prime}} \leq 1$ for $j^{\prime} \neq j$. Thus, the DESIs are also normalized to take values between 0 and 1 . Note also that

$$
\sigma_{j j^{\prime}}=\sigma_{j}+\sigma_{j^{\prime}}+v_{j j^{\prime}}, \quad j^{\prime} \neq j .
$$

Therefore, when $v_{j j^{\prime}}=0$, the DESI $\sigma_{j j^{\prime}}$ is simply the sum of the SESIs of the two biochemical factors $j$ and $j^{\prime}$.

Finally, it is worthwhile noticing that Eqs. (21), (33), and (39) imply that we can quantify the fractional contribution of all joint effects of order $\geq 3$ to the response variance by means of

$$
\gamma:=1-\sum_{j=1}^{J} \sigma_{j}-\sum_{j=1}^{J} \sum_{j^{\prime}>j}^{J} v_{j j^{\prime}} .
$$

When $\gamma \simeq 0$, these effects are negligible, in which case the use of indices $\sigma_{j}, \tau_{j}, \eta_{j}$, and $v_{j j^{\prime}}$ for sensitivity analysis will be sufficient. However, when $\gamma \neq 0$, we may want to investigate higher-order joint effects of triplets, quadruples, etc. To do so, we would have to evaluate higher-order sensitivity indices, which would require additional computational resources (memory and CPU time). For this reason, we limit our analysis to first- and second-order effects. We expect that in most cases of interest, these effects will provide a sufficient picture of the sensitivity properties of a biochemical reaction system.

We now summarize an algorithm for investigating the first- and second-order sensitivity properties of a biochemical reaction system by variance-based sensitivity analysis.

\section{A. Initialization}

1. Calculate the TESIs $\tau_{j}$ and the SESIs $\sigma_{j}$.

2. Calculate the JESIs $\eta_{j}=\tau_{j}-\sigma_{j}$.

3. Set a small threshold $\theta \ll 1$.

\section{B. Classification}

For $j=1,2, \ldots, J$ :

4. If $\eta_{j} \leq \theta$ and $\sigma_{j} \leq \theta$, conclude that the $j^{\text {th }}$ biochemical factor does not appreciably influence the system response.

5. If $\eta_{j} \leq \theta$ and $\sigma_{j}>\theta$, conclude that the $j^{\text {th }}$ biochemical factor influences the system response mostly singularly.

6. If $\eta_{j}>\theta$ and $\sigma_{j} \leq \theta$, conclude that the $j^{\text {th }}$ biochemical factor influences the system response mostly jointly with other factors.

7. If $\eta_{j}>\theta$ and $\sigma_{j}>\theta$, conclude that the $j^{\text {th }}$ biochemical factor influences the system response both singularly and jointly with other factors.

\section{Ranking}

8. Use the SESIs to rank the influential factors, with the most influential factor being the one with the largest SESI value, the second most influential factor being the one with the second largest value, and so on.

9. If desired, calculate the PESIs and DESIs. Use the PESIs to investigate the contribution to the response variance of biochemical factors that influence the system response jointly with another factor. Use the DESIs to rank pairs of biochemical factors, with the most influential pair being the one with the largest DESI value, the second most influential pair being the one with the second largest value, and so on.

\section{MONTE CARLO ESTIMATION}

The sensitivity indices derived from Sec. IV cannot be computed analytically. However, a number of numerical techniques are available for their evaluation, with the most prominent ones based on Monte Carlo simulation. ${ }^{23,26}$ In this section, we present a Monte Carlo method for estimating the variance-based sensitivity indices that uses a Latin hypercube sampling scheme $e^{38,44-46}$ to efficiently sample the random factors and reduce estimation variance. We will be referring to this technique as Monte Carlo Latin hypercube sampling (MC-LHS).

The MC-LHS method starts by forming two groups

$$
\begin{array}{cccc}
u_{1}^{(1)} & u_{2}^{(1)} & \cdots & u_{J}^{(1)} \\
u_{1}^{(2)} & u_{2}^{(2)} & \cdots & u_{J}^{(2)} \\
\vdots & \vdots & & \vdots \\
u_{1}^{(L)} & u_{2}^{(L)} & \cdots & u_{J}^{(L)} \\
u_{1}^{(L+1)} & u_{2}^{(L+1)} & \cdots & u_{J}^{(L+1)} \\
u_{1}^{(L+2)} & u_{2}^{(L+2)} & \cdots & u_{J}^{(L+2)} \\
\vdots & \vdots & & \vdots \\
u_{1}^{(2 L)} & u_{2}^{(2 L)} & \cdots & u_{J}^{(2 L)}
\end{array}
$$

of $2 L$ Latin hypercube samples of the statistically independent random factors $\mathbf{U}=\left\{U_{1}, U_{2}, \ldots, U_{J}\right\}$, where $L$ is a given 
sample size. ${ }^{47}$ The samples are drawn independently from the Gaussian probability densities of $U_{j}, j=1,2, \ldots, J$. In particular, when $U_{j}=Y_{j}^{\ddagger}$, the sample is drawn from a zeromean Gaussian distribution with standard deviation $\lambda_{j}^{\ddagger}$, whereas when $U_{j}=Y_{j}$, the sample is drawn from a zero-mean Gaussian distribution with standard deviation $\lambda_{j}$.

Subsequently, we group the samples together to form the following values for $\mathbf{U}$ :

$$
\begin{aligned}
\boldsymbol{u}^{(l)}= & \left\{u_{1}^{(l)}, u_{2}^{(l)}, \ldots, u_{J}^{(l)}\right\}, \quad l=1,2, \ldots, 2 L, \\
\boldsymbol{u}_{j}^{(l)}= & \left\{u_{1}^{(L+l)}, \ldots, u_{j-1}^{(L+l)}, u_{j}^{(l)}, u_{j+1}^{(L+l)}, \ldots, u_{J}^{(L+l)}\right\}, \\
& j=1,2, \ldots, J, \quad l=1,2, \ldots, L \\
\boldsymbol{u}_{(j)}^{(l)}= & \left\{u_{1}^{(l)}, \ldots, u_{j-1}^{(l)}, u_{j}^{(L+l)}, u_{j+1}^{(l)}, \ldots, u_{J}^{(l)}\right\} \\
& j=1,2, \ldots, J, \quad l=1,2, \ldots, L .
\end{aligned}
$$

We use these values together with Eq. (14) to determine the reaction rate constants of the biochemical reaction system and evaluate the corresponding $2 L(J+1)$ system responses $R\left(\boldsymbol{u}^{(l)}\right), R\left(\boldsymbol{u}_{j}^{(l)}\right)$, and $R\left(\boldsymbol{u}_{(j)}^{(l)}\right)$ by solving Eq. (2) and by using Eq. (4). Note, however, that before calculating each system response, we need to check whether the constraints on $\boldsymbol{U}$ imposed by Eq. (15) are satisfied. If they are not satisfied, then the corresponding value of $\boldsymbol{U}$ is rejected. We expect that for biophysically reasonable parameters (nominal rate constant values and standard chemical potential variances), the rejection rate will be small.

We use the evaluated responses to compute the following Monte Carlo estimators of the response variances: ${ }^{29}$

$$
\begin{aligned}
\widehat{\operatorname{Var}}_{j}[R(\boldsymbol{U})]= & \frac{1}{4 L}\left[\sum_{l=1}^{L} R^{2}\left(\boldsymbol{u}^{(l)}\right)+\sum_{l=1}^{L} R^{2}\left(\boldsymbol{u}^{(L+l)}\right)\right. \\
+ & \left.\sum_{l=1}^{L} R^{2}\left(\boldsymbol{u}_{j}^{(l)}\right)+\sum_{l=1}^{L} R^{2}\left(\mathbf{u}_{(j)}^{(l)}\right)\right]-\hat{E}_{j}^{2}[R(\boldsymbol{U})], \\
\widehat{\operatorname{Var}}_{j j^{\prime}}[R(\boldsymbol{U})]= & \frac{1}{4 L}\left[\sum_{l=1}^{L} R^{2}\left(\boldsymbol{u}_{j}^{(l)}\right)+\sum_{l=1}^{L} R^{2}\left(\boldsymbol{u}_{(j)}^{(l)}\right)\right. \\
& \left.+\sum_{l=1}^{L} R^{2}\left(\boldsymbol{u}_{j^{\prime}}^{(l)}\right)+\sum_{l=1}^{L} R^{2}\left(\boldsymbol{u}_{\left(j^{\prime}\right)}^{(l)}\right)\right] \\
& -\hat{E}_{j j^{\prime}}^{2}[R(\boldsymbol{U})], \\
\widehat{\operatorname{Var}}\left[E\left[R(\boldsymbol{U}) \mid U_{j}\right]\right]= & \frac{1}{2 L}\left[\sum_{l=1}^{L} R\left(\boldsymbol{u}^{(l)}\right) R\left(\boldsymbol{u}_{j}^{(l)}\right)\right. \\
& \left.+\sum_{l=1}^{L} R\left(\boldsymbol{u}^{(L+l)}\right) R\left(\boldsymbol{u}_{(j)}^{(l)}\right)\right]-\hat{E}_{j}^{2}[R(\boldsymbol{U})],
\end{aligned}
$$

$$
\begin{aligned}
\widehat{\operatorname{Var}}\left[E\left[R(\boldsymbol{U}) \mid \boldsymbol{U}_{(j)}\right]\right]= & \frac{1}{2 L}\left[\sum_{l=1}^{L} R\left(\boldsymbol{u}^{(l)}\right) R\left(\boldsymbol{u}_{(j)}^{(l)}\right)\right. \\
& \left.+\sum_{l=1}^{L} R\left(\boldsymbol{u}^{(L+l)}\right) R\left(\boldsymbol{u}_{j}^{(l)}\right)\right]-\hat{E}_{j}^{2}[R(\boldsymbol{U})],
\end{aligned}
$$

$$
\widehat{\operatorname{Var}}\left[E\left[R(\boldsymbol{U}) \mid U_{j}, U_{j^{\prime}}\right]\right]
$$

$$
\begin{aligned}
= & \frac{1}{2 L}\left[\sum_{l=1}^{L} R\left(\boldsymbol{u}_{j}^{(l)}\right) R\left(\boldsymbol{u}_{\left(j^{\prime}\right)}^{(l)}\right)+\sum_{l=1}^{L} R\left(\boldsymbol{u}_{j^{\prime}}^{(l)}\right) R\left(\boldsymbol{u}_{(j)}^{(l)}\right)\right] \\
& -\hat{E}_{j j^{\prime}}^{2}[R(\boldsymbol{U})],
\end{aligned}
$$

where

$$
\begin{aligned}
& \hat{E}_{j}[R(\boldsymbol{U})] \\
& \quad=\sqrt{\frac{1}{2 L}\left[\sum_{l=1}^{L} R\left(\boldsymbol{u}^{(l)}\right) R\left(\boldsymbol{u}^{(L+l)}\right)+\sum_{l=1}^{L} R\left(\boldsymbol{u}_{j}^{(l)}\right) R\left(\boldsymbol{u}_{(j)}^{(l)}\right)\right]}, \\
& \hat{E}_{j j^{\prime}}[R(\boldsymbol{U})] \\
& \quad=\sqrt{\frac{1}{2 L}\left[\sum_{l=1}^{L} R\left(\boldsymbol{u}_{j}^{(l)}\right) R\left(\boldsymbol{u}_{(j)}^{(l)}\right)+\sum_{l=1}^{L} R\left(\boldsymbol{u}_{j^{\prime}}^{(l)}\right) R\left(\boldsymbol{u}_{\left(j^{\prime}\right)}^{(l)}\right)\right]} .
\end{aligned}
$$

Finally, we estimate the variance-based sensitivity indices by [recall Eqs. (32), (33), (37), (40), and (41)]

$$
\begin{aligned}
& \hat{\tau}_{j}=1-\frac{\widehat{\operatorname{Var}}\left[E\left[R(\boldsymbol{U}) \mid \boldsymbol{U}_{(j)}\right]\right]}{\widehat{\operatorname{Var}_{j}}[R(\boldsymbol{U})]}, \\
& \hat{\sigma}_{j}=\frac{\widehat{\operatorname{Var}}\left[E\left[R(\boldsymbol{U}) \mid U_{j}\right]\right]}{\widehat{\operatorname{Var}_{j}}[R(\boldsymbol{U})]}, \\
& \hat{\eta}_{j}=\hat{\tau}_{j}-\hat{\sigma}_{j}, \\
& \hat{\sigma}_{j j^{\prime}}=\frac{\widehat{\operatorname{Var}}\left[E\left[R(\boldsymbol{U}) \mid U_{j}, U_{j^{\prime}}\right]\right]}{\widehat{\operatorname{Var}}_{j j^{\prime}}[R(\boldsymbol{U})]}, \quad j^{\prime} \neq j, \\
& \hat{v}_{j j^{\prime}}=\hat{\sigma}_{j j^{\prime}}-\hat{\sigma}_{j}-\hat{\sigma}_{j^{\prime}}, \quad j^{\prime} \neq j .
\end{aligned}
$$

The previous estimators are modified versions of the ones proposed previously by Saltelli. ${ }^{48}$ These modifications are important in order to guarantee that the estimated variances satisfy the following necessary conditions (for any number of Monte Carlo samples). ${ }^{29}$

C.1: $\widehat{\operatorname{Var}}_{j}[R(\boldsymbol{U})] \geq 0$ and $\widehat{\operatorname{Var}}_{j j^{\prime}}[R(\boldsymbol{U})] \geq 0$, 
C.2: If $U_{j}$ is fixed, then $\widehat{\operatorname{Var}}\left[E\left[R(\boldsymbol{U}) \mid U_{j}\right]\right]=0$

and $\widehat{\operatorname{Var}}\left[E\left[R(\boldsymbol{U}) \mid \boldsymbol{U}_{(j)}\right]\right]=\widehat{\operatorname{Var}_{j}}[R(\boldsymbol{U})]$,

C.3: If $\boldsymbol{U}_{(j)}$ is fixed, then $\widehat{\operatorname{Var}}\left[E\left[R(\boldsymbol{U}) \mid U_{j}\right]\right]=\widehat{\operatorname{Var}_{j}}[R(\boldsymbol{U})]$

and $\widehat{\operatorname{Var}}\left[E\left[R(\boldsymbol{U}) \mid \boldsymbol{U}_{(j)}\right]\right]=0$,

C.4: $\widehat{\operatorname{Var}}\left[E\left[R(\boldsymbol{U}) \mid U_{j}\right]\right]+\widehat{\operatorname{Var}}\left[E\left[R(\boldsymbol{U}) \mid \boldsymbol{U}_{(j)}\right]\right] \leq \widehat{\operatorname{Var}}_{j}[R(\boldsymbol{U})]$,

C.5: If $U_{j}$ and $U_{j^{\prime}}$ are fixed, then $\widehat{\operatorname{Var}}\left[E\left[R(\boldsymbol{U}) \mid U_{j}, U_{j^{\prime}}\right]\right]=0$,

C.6: If $\boldsymbol{U}_{\left(j, j^{\prime}\right)}$ is fixed, then $\widehat{\operatorname{Var}}\left[E\left[R(\boldsymbol{U}) \mid U_{j}, U_{j^{\prime}}\right]\right]$

$=\widehat{\operatorname{Var}}_{j j^{\prime}}[R(\boldsymbol{U})]$,

C.7: $\widehat{\operatorname{Var}}\left[E\left[R(\boldsymbol{U}) \mid U_{j}, U_{j^{\prime}}\right]\right]+\widehat{\operatorname{Var}}\left[E\left[R(\boldsymbol{U}) \mid \boldsymbol{U}_{\left(j, j^{\prime}\right)}\right]\right]$

$\leq \widehat{\operatorname{Var}}_{j j^{\prime}}[R(\boldsymbol{U})]$

Note that in Conditions C.6 and C.7, $\boldsymbol{U}_{\left(j, j^{\prime}\right)}$ is the set of all factors excluding $U_{j}$ and $U_{j^{\prime}}$.

Condition C.1 guarantees that the two estimators for the response variance are non-negative. Estimators for the conditional response variances $\operatorname{Var}\left[E\left[R(\boldsymbol{U}) \mid U_{j}\right]\right]$, $\operatorname{Var}\left[E\left[R(\boldsymbol{U}) \mid \boldsymbol{U}_{(j)}\right]\right]$, and $\operatorname{Var}\left[E\left[R(\boldsymbol{U}) \mid U_{j}, U_{j^{\prime}}\right]\right]$ must also be non-negative. However, this is not necessarily true for the previous estimators. We could derive non-negative variance estimators by employing the standard Monte Carlo formulas

$$
\begin{aligned}
& \widehat{\operatorname{Var}}[W]=\frac{1}{L} \sum_{l=1}^{L}\left(w^{(l)}-\hat{E}[W]\right)^{2}, \text { where } \\
& \hat{E}[W]=\frac{1}{L} \sum_{l=1}^{L} w^{(l)},
\end{aligned}
$$

but using these formulas results in an unnecessarily large number of system response evaluations. ${ }^{48}$ Note, however, that if the variance estimators are non-negative, then Condition C.4 guarantees that $0 \leq \hat{\sigma}_{j} \leq 1,0 \leq \hat{\tau}_{j} \leq 1$, and $\hat{\sigma}_{j} \leq \hat{\tau}_{j}$ as expected. This implies that $0 \leq \hat{\eta}_{j}:=\hat{\tau}_{j}-\hat{\sigma}_{j} \leq 1$. Moreover, Condition C.7 guarantees that $0 \leq \hat{\sigma}_{j j^{\prime}} \leq 1$, which also implies that $\hat{v}_{j j^{\prime}} \leq 1$. On the other hand, Conditions C.2 and C.3 guarantee that when $U_{j}$ is fixed, then $\hat{\sigma}_{j}=\hat{\tau}_{j}=0$, whereas when $\boldsymbol{U}_{(j)}$ is fixed, then $\hat{\sigma}_{j}=\hat{\tau}_{j}=1$. Finally, Conditions C.5 and C.6 guarantee that when $U_{j}$ and $U_{j^{\prime}}$ are fixed, then $\hat{\sigma}_{j j^{\prime}}=0$, whereas when $\boldsymbol{U}_{\left(j, j^{\prime}\right)}$ is fixed, then $\hat{\sigma}_{j j^{\prime}}=1$. In our experience, by using the previous estimators, instead the ones suggested in Ref. 48, we obtain a more efficient numerical implementation of the sensitivity analysis methodology discussed in Sec. IV, which, with fewer Monte Carlo samples, produces estimates of the underlying sensitivity indices consistent with all necessary conditions and constraints.

\section{CASE STUDY: THE MITOGEN-ACTIVATED PROTEIN KINASE CASCADE}

To illustrate the previous variance-based sensitivity analysis technique, we now focus our attention on a specific biochemical reaction system that models the well-known MAPK signaling cascade. The MAPK cascade is an important signaling pathway that couples the binding of growth factors to cell surface receptors with intracellular responses that control cellular growth, proliferation, differentiation, and survival. ${ }^{49}$ We use a rather detailed model of this pathway, depicted in Fig. 2, introduced in the literature by Schoeberl et al. ${ }^{50}$ This model consists of $N=23$ molecular species that interact with each other through $M=21$ reactions (11 reactions are reversible, whereas the remaining ten reactions are irreversible). For simplicity, we have removed all reactions that characterize signal transduction from the epidermal growth factor receptor to Ras-GTP, which is the input to the MAPK cascade.

The MAPK model begins with the synthesis of RasGTP, which interacts with Raf kinase to produce an active version Raf* of Raf; see Fig. 2. Raf* is capable of producing a doubly phosphorylated active version MEK-PP of the kinase MEK by two successive phosphorylation steps, whereas MEK-PP can produce a doubly phosphorylated active version ERK-PP of the extracellular signal-regulated kinase (ERK) by two phosphorylation events as well. Each phosphorylation step is considered to be irreversible, unless mediated by an inactivating phosphatase. In this case, Phol, Pho2, and Pho3 model inactivating phosphatases for Raf, MEK, and ERK, respectively.

In the accompanying supplementary material, ${ }^{29}$ we have summarized the reactions associated with the MAPK cascade model and listed the nominal values of the associated normalized reaction rate constants as well as the initial activities of the molecular species involved. We have also shown that the chosen values for the reaction rate constants lead to a thermodynamically feasible model. Note that for an irreversible reaction, we set the rate constant of the corresponding reverse reaction equal to zero.

We will focus our sensitivity analysis effort on the activity profile of ERK-PP, which is the output of the MAPK cascade; see Fig. 2(d) of the supplementary material. ${ }^{29} \mathrm{We}$ adopt the logarithms of the timing, duration, and strength of this profile as the response characteristics of interest for sensitivity analysis. We consider three strategies for sensitivity analysis: (a) reaction-oriented sensitivity analysis (ROSA), (b) species-oriented sensitivity analysis (SOSA), and (c) reaction-oriented/species-oriented sensitivity analysis (ROSOSA). ROSA investigates only the effects of fluctuations in the standard chemical potentials of the activated complexes, whereas SOSA investigates only the effects of fluctuations in the standard chemical potentials associated with the molecu- 


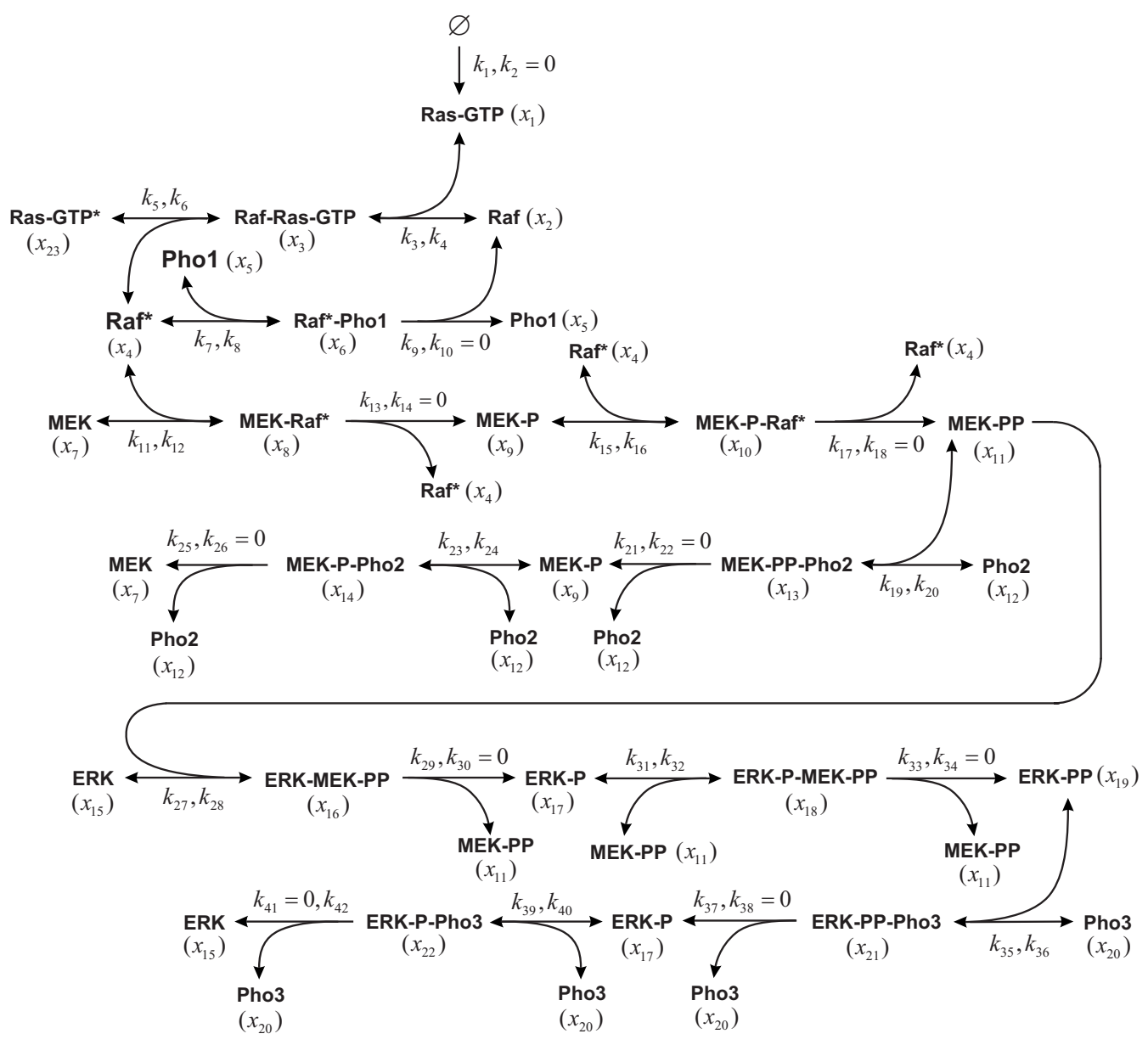

FIG. 2. A biochemical reaction model of the MAPK signaling cascade.

lar species. On the other hand ROSOSA investigates the sensitivity behavior of a biochemical reaction system under both types of fluctuations.

ROSA and SOSA can be useful in drug-design problems in which pharmacological control of the response characteristics of a biochemical reaction system is of interest. ROSA can be used when the objective is to modify the response of a biochemical reaction system by pharmacologically targeting selected enzymes responsible for catalyzing influential reactions, whereas SOSA can be used when the objective is to modify the system response by altering the kinetic properties of selected influential molecular species. On the other hand, ROSOSA provides a more general approach to sensitivity analysis. As a matter of fact, we can use ROSOSA to obtain a complete picture of the sensitivity properties of a biochemical reaction system both with respect to the underlying reactions and reactant molecular species. In this section, we illustrate the use of ROSOSA for identifying the reaction rate constants responsible for influencing the system response. This task is important in reverse engineering problems, where the objective is to estimate the reaction rate constants of a biochemical reaction system from available data. Use of ROSOSA may help us to focus our estimation effort on "influential" reaction rate constants, whose values must be determined with high accuracy, and ignore the remaining "noninfluential" reaction rate constants, whose exact values are of no particular interest.
Although, in general, the standard deviations associated with the standard chemical potentials of the activated complexes and molecular species depend on $m$ and $n$, respectively, this dependence may not be useful in practice, since it is difficult to obtain information about the fluctuation levels of these quantities. For this reason, we assume here that $\lambda_{m}^{\ddagger}$ $=\lambda^{\ddagger}, \lambda_{n}=\lambda$, and consider $\lambda^{\ddagger}$ and $\lambda$ as two "user-defined" parameters that control the "scale" of sensitivity analysis. Small values of $\lambda^{\ddagger}$ and $\lambda$ correspond to "local" sensitivity analysis associated with small fluctuations in the standard chemical potentials about their nominal values, whereas large values of $\lambda^{\#}$ and $\lambda$ correspond to "global" sensitivity analysis associated with large fluctuations in the standard chemical potentials. Note that even under this simplification, when using SOSA (in which case $\lambda^{\ddagger}=0, \lambda \neq 0$ ) or ROSOSA (in which case $\lambda^{\ddagger}, \lambda \neq 0$ ), the size of variations applied on the reaction rates will not be uniform due to Eq. (17).

In this section, we investigate the sensitivity properties of MAPK by implementing the classification/ranking steps presented in Sec. IV with threshold $\theta=0.1$ for the ROSA and SOSA and $\theta=0.05$ for the ROSOSA, which corresponds to $10 \%$ and $5 \%$ of the maximum attainable JESI and SESI values, respectively. We take the ROSOSA threshold value to be half of the one used in ROSA and SOSA, since, in the former case, the response variance is distributed among two types of biochemical factors (i.e., among the standard chemical potentials of the activated complexes and the standard chemical 
ROSA
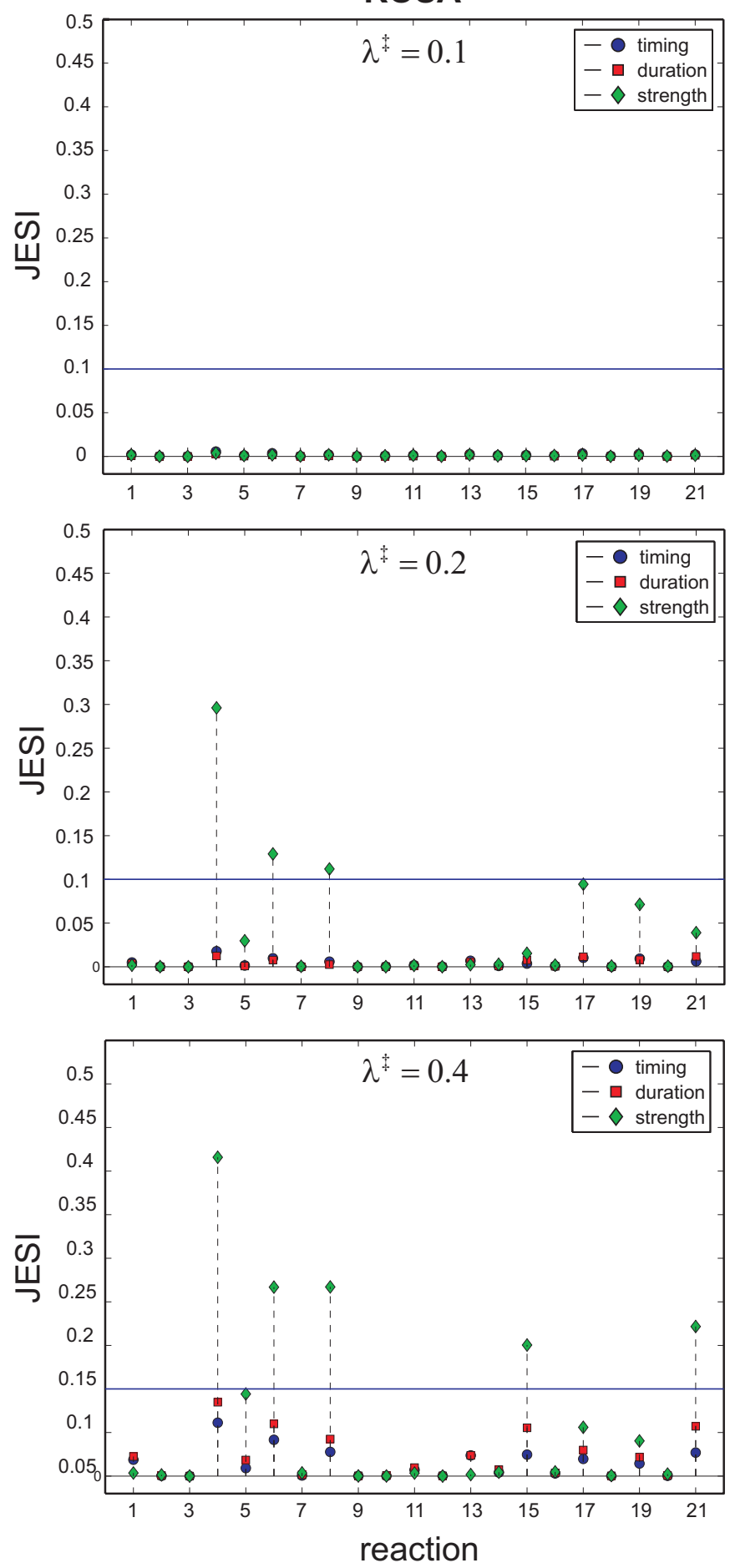

ROSA
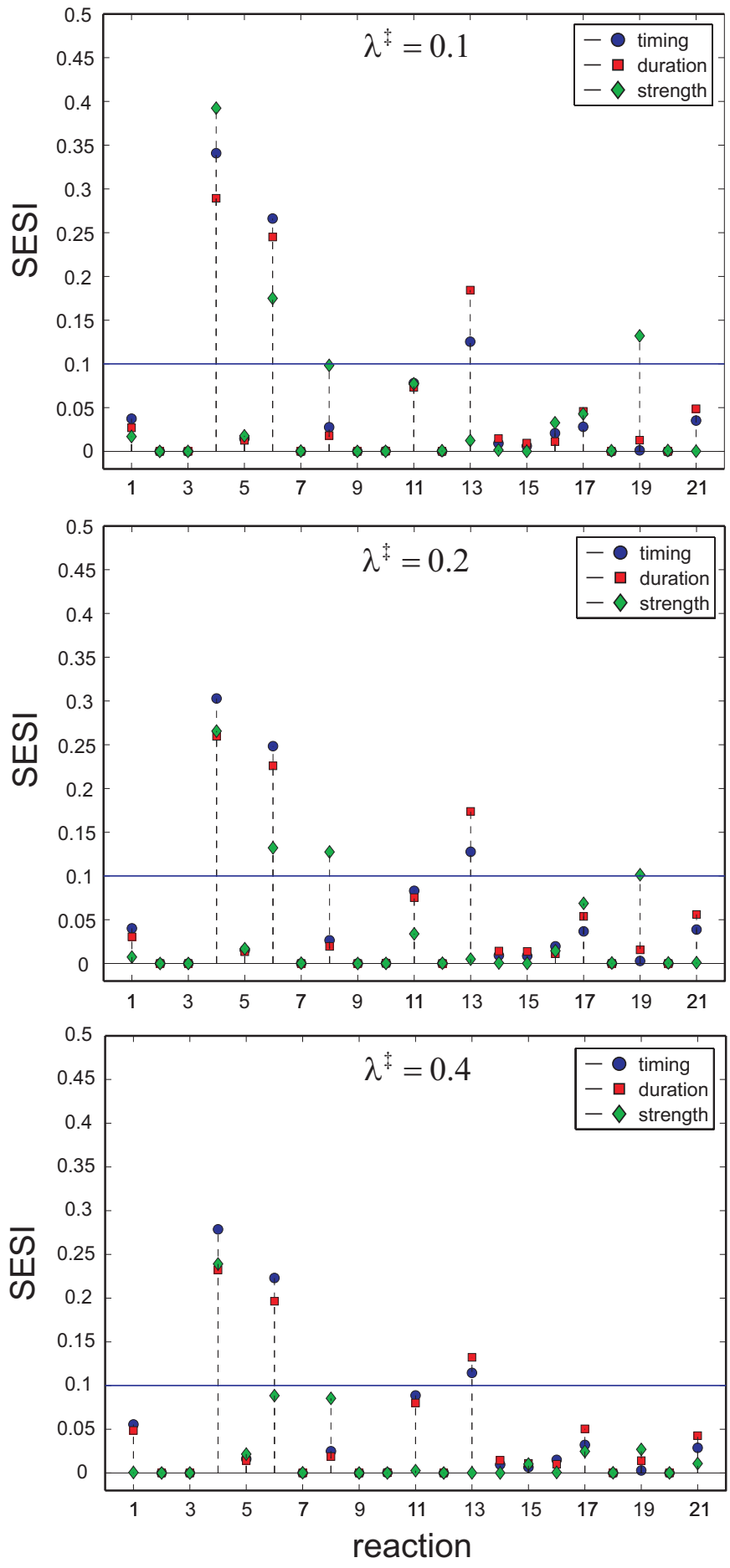

FIG. 3. ROSA results for the MAPK cascade at three different fluctuation levels with $\lambda^{\ddagger}=0.1,0.2,0.4$.

potentials of the molecular species), as opposed to the latter cases in which the response variance is distributed among only one type of biochemical factors. Choosing a threshold value is a relatively easy task in variance-based sensitivity analysis techniques, since the indices are normalized to take values between 0 and 1 . In the following, we estimate the variance-based sensitivity indices associated with the timing, duration, and strength of the ERK-PP activity profile, as defined by Eq. (4). We do this by considering the dynamic behavior of MAPK within a time frame of $6 \mathrm{~h}\left(t_{\max }\right.$
$=360 \mathrm{~min}$ ), by setting $\varepsilon=0.05$ in Eq. (5), and by employing the MC-LHS estimators presented in Sec. V with $L=3000$.

In Fig. 3, we depict the ROSA results for the MAPK cascade at three different fluctuation levels of the standard chemical potentials of the activated complexes. ${ }^{51}$ In the case of timing and duration, the estimated values of $\gamma$ turn out to be all zero, which implies that there is no appreciable fractional contribution to the response variances from high-order $(\geq 3)$ joint effects. The same is true in the case of strength with $\lambda^{\ddagger}=0.1$. However, when $\lambda^{\ddagger}=0.2,0.4$, the estimated $\gamma$ 
values are 0.0713 and 0.1534 , respectively, which indicate emergence of high-order $(\geq 3)$ joint effects for larger values of $\lambda^{\ddagger}$. As a matter of fact, when $\lambda^{\ddagger}=0.2$, these joint effects account for about $7 \%$ of the response variance, whereas when $\lambda^{\ddagger}=0.4$, they account for about $15 \%$ of the response variance. A closer look at the results depicted in Fig. 3 indicates that only the strength may be subject to second-order joint effects, since, when $\lambda^{\ddagger}=0.2,0.4$, some JESI values associated with this response characteristic are above the threshold. As a matter of fact most estimated values of $\delta$ are small $(\delta \leq 0.1340)$, except the values associated with the strength when $\lambda^{\ddagger}=0.2,0.4$, which have been estimated to be 0.2233 and 0.4883 , respectively.

In all three cases depicted in Fig. 3, the timing and duration are singularly influenced by the same three reactions 4 (Raf*+Pho1 $\rightleftarrows$ Raf*-Pho1), 6 (MEK+Raf* $\rightleftarrows$ MEK-Raf*), and 13 (MEK $-\mathrm{P}-\mathrm{Pho} 2 \rightarrow \mathrm{MEK}+\mathrm{Pho} 2)$, with reaction 4 being the most influential and reaction 13 being the least influential. As a matter of fact, the estimated SESI values indicate that these three reactions account for about $72 \%, 67 \%$, and $60 \%$ of the timing or duration variance when $\lambda^{\ddagger}=0.1,0.2$, and 0.4, respectively. However, the results depicted in Fig. 3 indicate a more complex sensitivity behavior for the strength.

When $\lambda^{\ddagger}=0.1$, the ROSA results indicate that the strength is influenced singularly by reaction 4 (Raf* +Pho1 $\rightleftarrows$ Raf $^{*}-$ Pho1), reaction $6 \quad$ (MEK+Raf* $\rightleftarrows$ MEK $\left.-\mathrm{Raf}^{*}\right)$, and reaction $19 \quad(\mathrm{ERK}-\mathrm{PP}-\mathrm{Pho} 3 \rightarrow$ ERK-P + Pho3), with reaction 4 being the most influential and reaction 19 being the least influential. As a matter of fact, the estimated SESI values indicate that these three reactions account for about $70 \%$ of the strength variance.

When $\lambda^{\star}=0.2$, the strength is being influenced both singularly and jointly by reaction 4 Raf $^{*}+\mathrm{Pho} 1 \rightleftarrows \mathrm{Raf}^{*}$ -Pho1), by reaction 6 (MEK $+\mathrm{Raf}^{*} \rightleftarrows \mathrm{MEK}-\mathrm{Raf}^{*}$ ), and by reaction 8 (MEK-P+Raf* $\left.\rightleftarrows \mathrm{MEK}-\mathrm{P}-\mathrm{Raf}^{*}\right)$, whereas reaction 19 (ERK-PP-Pho3 $\rightarrow$ ERK $-\mathrm{P}+\mathrm{Pho} 3)$ still influences the strength singularly, although the corresponding SESI value has now been reduced, being above the threshold only marginally. Inspection of the estimated PESI values (data not shown) reveals that the pairwise influence of reaction 4 on the strength is mostly with reactions 5,8 , and 17 , whereas reaction 8 primarily influences the strength jointly with reaction 21. It turns out that these singular and joint effects account for about $70 \%$ of the strength variance, with $76 \%$ of this amount being attributed to singular and joint effects among reactions 4,6 , and 8 . On the other hand, inspection of the estimated DESI values (data not shown) reveals that pairs (4-6), (4-8), and (6-8) account for about 40\%, 40\%, and $27 \%$ of the strength variance, respectively.

Finally, when $\lambda^{\ddagger}=0.4$, reaction 19 does not influence the strength anymore. In this case, the strength is influenced both singularly and jointly only by reaction 4 (Raf* + Pho1 $\rightleftarrows$ Raf $^{*}-$ Pho1), with reaction 6 (MEK $\left.+\mathrm{Raf}^{*} \rightleftarrows \mathrm{MEK}-\mathrm{Raf}^{*}\right)$, reaction 8 (MEK-P+Raf* $\rightleftarrows \mathrm{MEK}$ $\left.-\mathrm{P}-\mathrm{Raf}^{*}\right)$, reaction $21(\mathrm{ERK}-\mathrm{P}-\mathrm{Pho} 3 \rightarrow \mathrm{ERK}+\mathrm{Pho} 3)$, and reaction 15 (ERK-MEK $-\mathrm{PP} \rightarrow \mathrm{ERK}-\mathrm{P}+\mathrm{MEK}-\mathrm{PP})$ influencing the strength only jointly. Inspection of the estimated SESI values and the estimated PESI values (data not shown) reveals that these singular and joint effects account for about
TABLE II. MAPK ROSA results.

\begin{tabular}{|c|c|c|c|c|}
\hline No. & Reaction & $T$ & $D$ & $S$ \\
\hline 4 & $\mathrm{Raf}^{*}+\mathrm{Pho} 1 \rightleftarrows \mathrm{Raf}^{*}-\mathrm{Pho} 1$ & - & • & - \\
\hline 6 & $\mathrm{MEK}+\mathrm{Raf}^{*} \rightleftarrows \mathrm{MEK}-\mathrm{Raf}^{*}$ & • & • & • \\
\hline 8 & $\mathrm{MEK}-\mathrm{P}+\mathrm{Raf}^{*} \rightleftarrows \mathrm{MEK}-\mathrm{P}-\mathrm{Raf}^{*}$ & & & • \\
\hline 13 & $\mathrm{MEK}-\mathrm{P}-\mathrm{Pho} 2 \rightarrow \mathrm{MEK}+\mathrm{Pho} 2$ & • & • & \\
\hline
\end{tabular}

$72 \%$ of the strength variance, with $73 \%$ of this amount being attributed to singular and joint effects among reactions 4,6 , and 8. On the other hand, inspection of the estimated DESI values (data not shown) reveals that pairs (4-8), (4-6), and (6-8) account for about $42 \%, 35 \%$, and $18 \%$ of the strength variance, respectively.

As a consequence of the previous results, we may conclude that the timing and duration are being influenced by reactions 4,6 , and 13 , whereas the strength is predominantly influenced by reactions 4,6 , and 8 ; see Table II.

In Fig. 4, we depict the SESI values evaluated by means of Eq. (34), with the response derivatives being approximated by symmetric finite differences around the nominal reaction rate values, as well as the SESI values estimated by MC-LHS. Note that Eq. (34) provides a reasonable approximation of the estimated MC-LHS SESI values when $\lambda^{\sharp}=0.1$. The derivative-based SESI values correctly predict that when $\lambda^{\ddagger}=0.1$, reactions 4,6 , and 13 are influential for the timing and duration, whereas reactions 4, 6, and 19 are influential for the strength. They also predict that reactions 4 , 6 , and 13 account for $74.68 \%$ of the timing and $73.12 \%$ of the duration variance, as compared to $73.24 \%$ and $71.87 \%$ predicted by MC-LHS, respectively. Moreover, these values predict that reactions 4,6 , and 19 account for $68.60 \%$ of the strength variance, as compared to $69.93 \%$ predicted by MCLHS. However, it is clear from the results depicted in Fig. 4 that the accuracy of the derivative-based SESI values decreases as $\lambda^{\ddagger}$ increases. As a matter of fact, the prediction that reactions 4,6 , and 13 account for $73.12 \%$ of the duration variance is inaccurate when compared to $65.93 \%$ and $56.09 \%$ predicted by MC-LHS for $\lambda^{\ddagger}=0.2$ and $\lambda^{\ddagger}=0.4$, respectively. The same is true for the timing and strength variances. Moreover, by using the derivative-based SESI values, we cannot detect the diminishing role of reaction 19 and the emergence of reaction 8 as a key influential factor for the strength. These deficiencies are expected, since the derivative-based SESI approximation, given by Eq. (34), cannot account for joint effects, which become prominent at increasing levels of standard chemical potential fluctuations. Therefore, it becomes clear that special care should be exercised when employing the approximation given by Eq. (34) for sensitivity analysis, whose use must be limited to problems with negligible joint effects. ${ }^{52}$

In Fig. 5, we depict the SOSA results at three different fluctuation levels of the standard chemical potentials of the molecular species. In the case of timing and duration, the estimated values of $\gamma$ turn out to be small $(\gamma \leq 0.0354)$, which implies that there is no appreciable fractional contribution to the response variances from high-order $(\geq 3)$ joint effects. The same is true in the case of strength with $\lambda=0.1$. 
ROSA
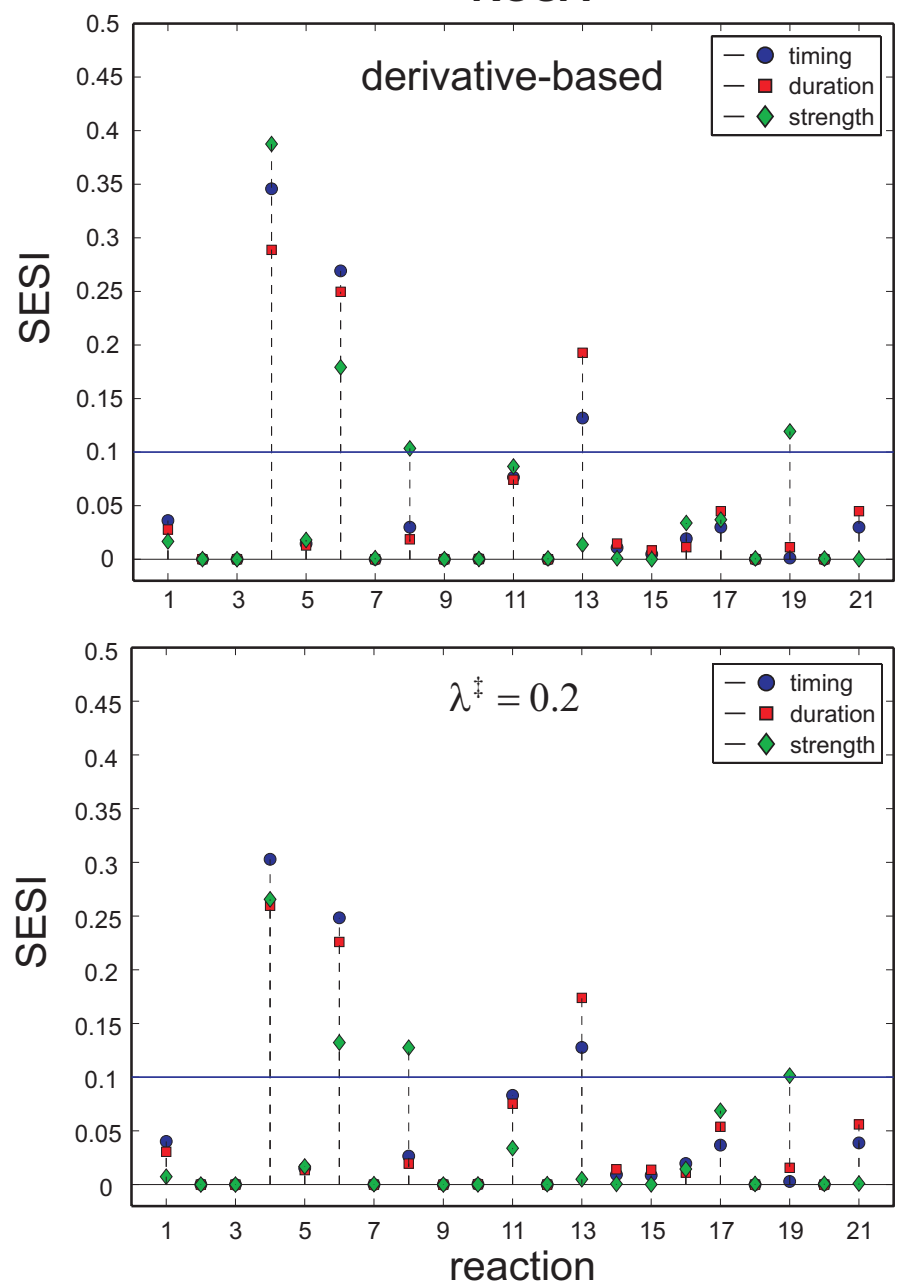

ROSA
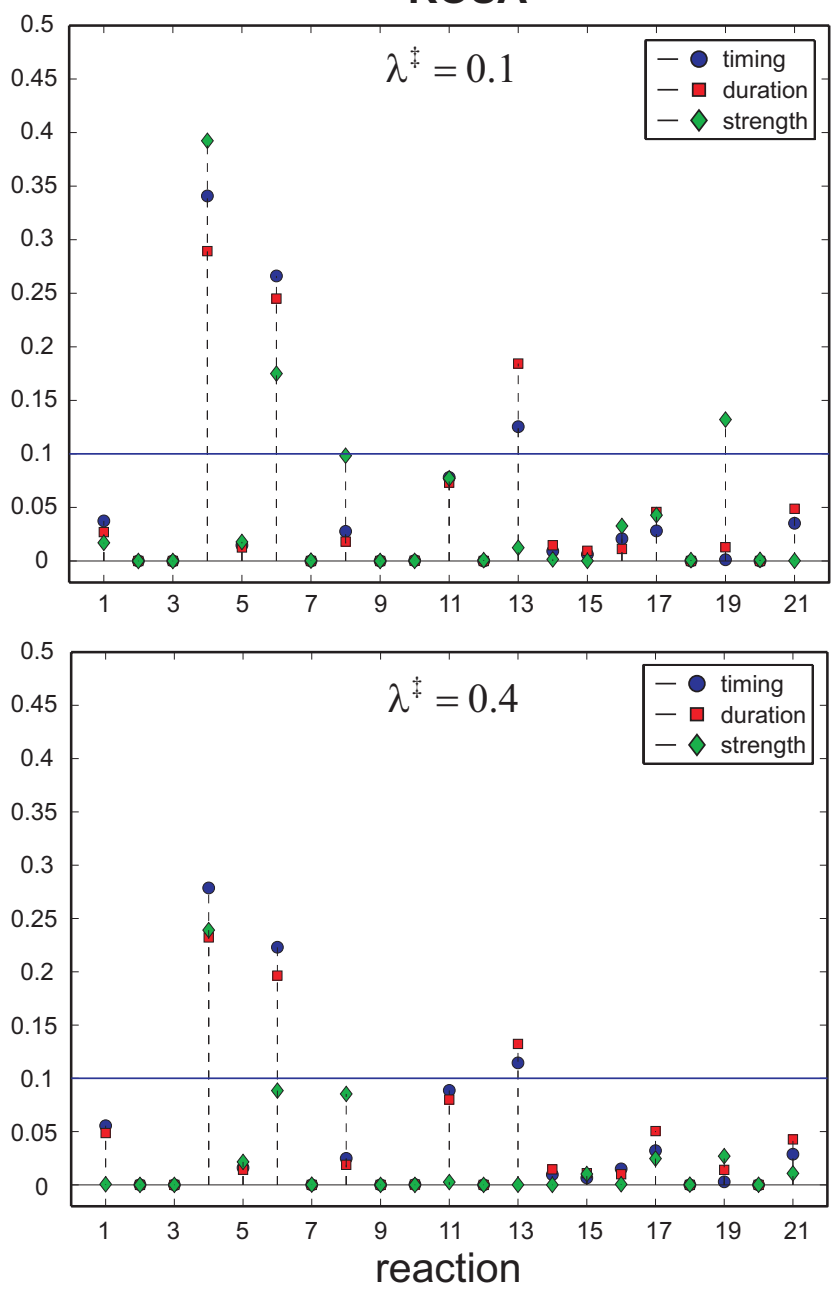

FIG. 4. Derivative-based vs MC-LHS estimation of SESI values associated with ROSA when $\lambda^{\ddagger}=0.1,0.2,0.4$.

However, when $\lambda=0.2,0.4$, the estimated $\gamma$ values are 0.0585 and 0.1271 , respectively, which indicate emergence of high-order $(\geq 3)$ joint effects for larger values of $\lambda$. As a matter of fact, when $\lambda=0.2$, these joint effects account for about $6 \%$ of the response variance, whereas when $\lambda=0.4$, they account for about $13 \%$ of the response variance. A closer look at the results indicates that the duration and strength may be subject to second-order joint effects, since, when $\lambda=0.4$, a JESI value associated with the duration is above the threshold, whereas, several JESI values associated with the strength are above the threshold when $\lambda=0.2,0.4$. As a matter of fact, most estimated values of $\delta$ are small, except the values associated with the duration when $\lambda=0.4$ and strength when $\lambda=0.2,0.4$, which have been estimated to be $0.1694,0.2905$, and 0.4045 , respectively.

In all three cases depicted in Fig. 5, the timing and duration are singularly influenced by the same three molecular species, 5 (Pho1), 7 (MEK), and 14 (MEK-P-Pho2), with species 5 being the most influential and species 14 being the least influential. As a matter of fact, the estimated SESI values indicate that these three species account for about $80 \%$, $77 \%$, and $67 \%$ of the timing or duration variance when $\lambda=0.1,0.2$, and 0.4 , respectively. When $\lambda=0.4$, the duration is influenced by species 5 both singularly and jointly. Inspec- tion of the estimated PESI values (data not shown) reveals that the pairwise influence of species 5 on the duration is mostly with species 9 (MEK-P), 14, 18 (ERK-P-MEK-PP), and 22 (ERK-P-Pho3). However, this joint influence contributes only $5.7 \%$ of the duration variance. On the other hand, inspection of the estimated DESI values (data not shown) reveals that pairs $(5-7),(5-14)$, and (7-14) account for about $49 \%, 47 \%$, and $33 \%$ of the duration variance, respectively.

When $\lambda=0.1$, the strength is being influenced singularly by species 5 (Pho1), 9 (MEK-P), and 7 (MEK), with species 5 being the most influential and species 7 being the least influential. The estimated SESI values indicate that these three species account for $81 \%$ of the strength variance.

When $\lambda=0.2$, the strength is being influenced both singularly and jointly by species 5 and 9 , whereas species 7 influences the strength only jointly. Inspection of the estimated PESI values (data not shown) reveals that the pairwise influence of species 5 on the strength is mostly with species 7, 9, and 21. Moreover, species 9 influences the strength jointly with species 21 , whereas species 7 influences the strength jointly with species 18 . It turns out that these singular and joint effects account for $77 \%$ of the strength variance, with $95 \%$ of this amount being attributed to singular and 


\section{SOSA}
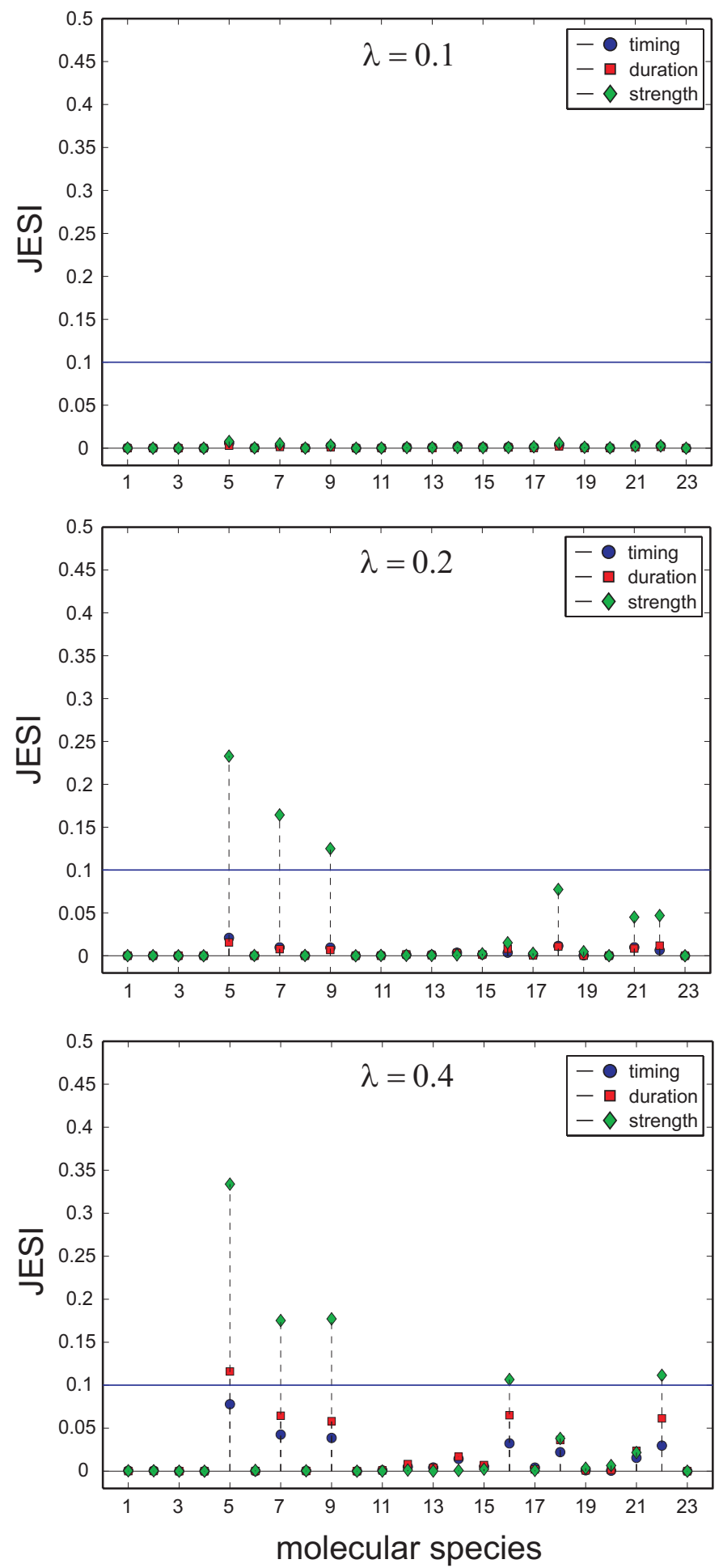

\section{SOSA}
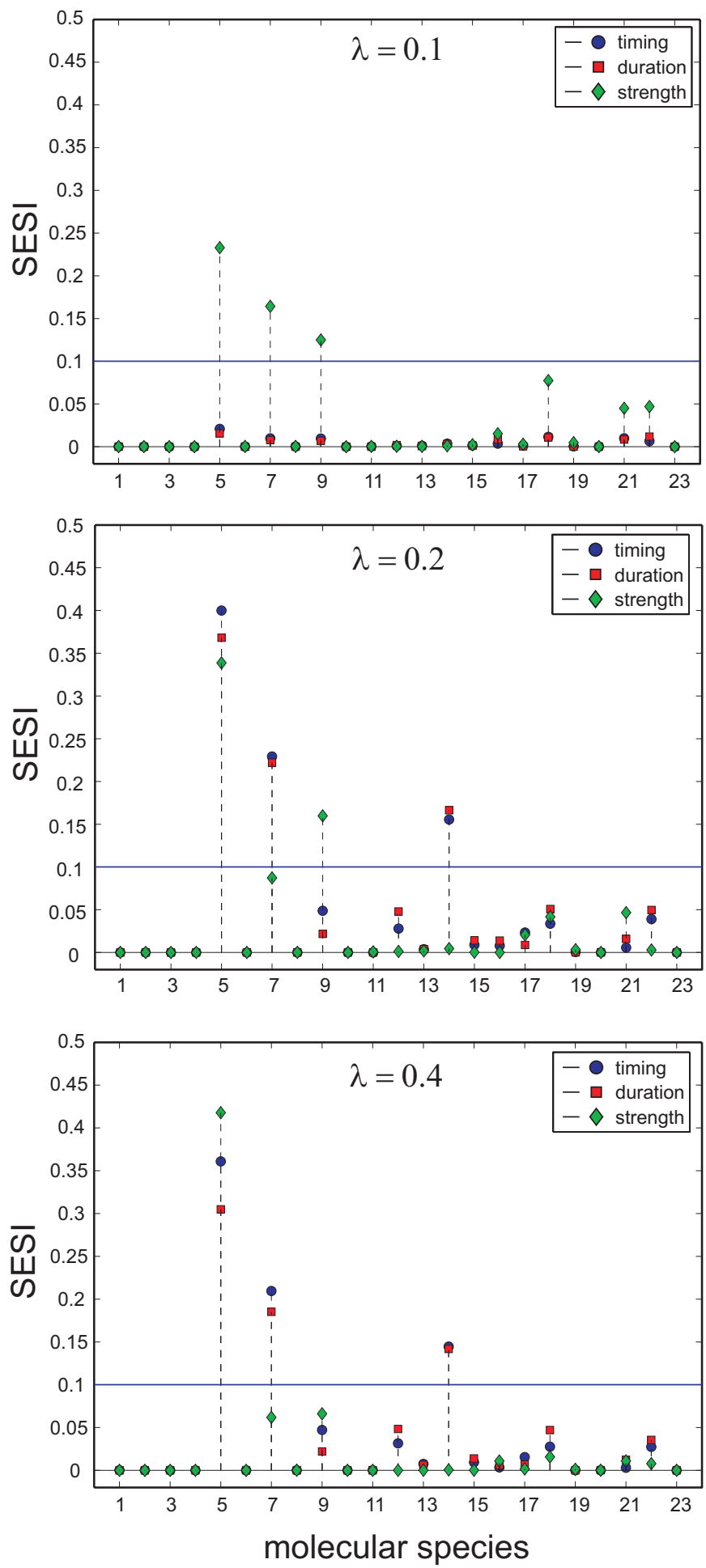

FIG. 5. SOSA results for the MAPK cascade at three different fluctuation levels with $\lambda=0.1,0.2,0.4$.

joint effects among species 5, 7, and 9. On the other hand, inspection of the estimated DESI values (data not shown) reveals that pairs (5-9), (5-7), and (7-9) account for about $57 \%, 50 \%$, and $22 \%$ of the strength variance, respectively.

Finally, when $\lambda=0.4$, the strength is being influenced both singularly and jointly only by species 5 , with species 9 , 7, 16 (ERK-MEK-PP), and 22 (ERK-P-Pho3) influencing the strength only jointly. Inspection of the estimated PESI values (data not shown) reveals that the pairwise influence of species 5 on the strength is mostly with species 7, 9, 16 (ERKMEK-PP), and 22 (ERK-P-Pho3), whereas species 7 influences the strength jointly with species 9 and with species 22 . It turns out that these singular and joint effects account for about $77 \%$ of the strength variance, with the singular and joint effects among species 5,9 , and 7 accounting for about $89 \%$ of that amount. Inspection of the estimated DESI values 
TABLE III. MAPK SOSA results.

\begin{tabular}{rcccc}
\hline \hline & $\begin{array}{c}\text { Molecular } \\
\text { No. }\end{array}$ & $T$ & $D$ & $S$ \\
\hline 5 & Phocies & $\bullet$ & $\bullet$ & $\bullet$ \\
7 & MEK & $\bullet$ & $\bullet$ & $\bullet$ \\
9 & MEK-P & & & $\bullet$ \\
\hline \hline
\end{tabular}

(data not shown) reveals that pairs (5-9), (5-7), and (7-9) account for about $56 \%, 52 \%$, and $16 \%$ of the strength variance, respectively.

As a consequence of the previous results, we may conclude that the timing and duration are being influenced by species 5,7 , and 14 , whereas the strength is predominantly influenced by species 5, 7, and 9; see Table III.

The results obtained by ROSOSA with $\lambda^{*}=\lambda=0.1$, $0.2,0.4$ perfectly agree with the previous conclusions and reveal no additional sensitivity behavior (data not shown). However, we can also use ROSOSA to identify the most influential reaction rates in a biochemical reaction system. Indeed, from Eq. (14), note that the reaction rate constants $K_{2 m-1}$ and $K_{2 m}$ of the $m^{\text {th }}$ forward and reverse reactions are influenced by the biochemical factors $U_{m}$ and $U_{M+n}$, $n=1,2, \ldots, N$, through the zero-mean Gaussian random variables $G_{m}$ and $G_{m}^{\prime}$, respectively, given by Eq. (16). As a consequence, we can quantify the influence of the rate constants $K_{2 m-1}$ and $K_{2 m}$ on the system response by means of the net fractional contribution of the biochemical factors $U_{m}$ and $U_{M+n}, n=1,2, \ldots, N$, on the system response. This leads to the following sensitivity indices:

$$
\begin{aligned}
g_{2 m-1}= & \sigma_{m}+\sum_{n=1}^{N} \operatorname{sgn}\left(\nu_{n m}\right) \sigma_{n}+\sum_{n=1}^{N} \operatorname{sgn}\left(\nu_{n m}\right) v_{m n} \\
& +\sum_{n=1}^{N} \sum_{n^{\prime}=n+1}^{N} \operatorname{sgn}\left(\nu_{n m}\right) \operatorname{sgn}\left(\nu_{n^{\prime} m}\right) v_{n n^{\prime}}, \\
g_{2 m}= & \sigma_{m}+\sum_{n=1}^{N} \operatorname{sgn}\left(\nu_{n m}^{\prime}\right) \sigma_{n}+\sum_{n=1}^{N} \operatorname{sgn}\left(\nu_{n m}^{\prime}\right) v_{m n} \\
& +\sum_{n=1}^{N} \sum_{n^{\prime}=n+1}^{N} \operatorname{sgn}\left(\nu_{n m}^{\prime}\right) \operatorname{sgn}\left(\nu_{n^{\prime} m}^{\prime}\right) v_{n n^{\prime}},
\end{aligned}
$$

where $\sigma_{m}$ is the SESI of the $m^{\text {th }}$ reaction, $\sigma_{n}$ is the SESI of the $n^{\text {th }}$ species, $v_{m n}$ is the PESI between reaction $m$ and species $n, v_{n n^{\prime}}$ is the PESI between species $n$ and $n^{\prime}$, and $\operatorname{sgn}(\cdot)$ is the sign function [i.e., $\operatorname{sgn}(a)=1$ if $a>0$, whereas $\operatorname{sgn}(a)$ $=0$ if $a=0]$. We refer to $g_{2 m-1}$ and $g_{2 m}$ as the group-effect sensitivity index (GESI) of the forward and reverse $m^{\text {th }}$ reactions, respectively. By using these indices, we can say that a reaction rate is most influential if the corresponding GESI value is the largest one. Likewise, we may define the second most influential rate constant, and so on.

In Fig. 6, we depict the ROSOSA results for the reaction rate constants at three different fluctuation levels of the standard chemical potentials. It is clear from these results that only a small fraction of the reaction rate constants apprecia- bly influence the timing, duration, and strength of ERK-PP. We summarize these reactions in Table IV, which depicts only the reaction rates which, for a given response characteristic, are consistently classified as being influential at all three fluctuation levels. The results also indicate that the timing and duration are influenced by the same reaction rate constants, namely, $\kappa_{7}, \kappa_{8}, \kappa_{11}, \kappa_{12}, \kappa_{24}$, and $\kappa_{25}$. Four of these rate constants, namely, $\kappa_{7}, \kappa_{8}, \kappa_{11}$, and $\kappa_{12}$, influence the strength as well, which is also influenced by $\kappa_{15}$ and $\kappa_{23}$. The most influential rate constants for the timing and duration are $\kappa_{7}$ and $\kappa_{11}$, whereas $\kappa_{7}$ is the most influential rate constant for the strength.

\section{FURTHER DISCUSSION}

Our ROSA and ROSOSA sensitivity analysis results summarized in Tables II and IV indicate that the binding and unbinding of the active version Raf* of the Raf kinase with its inactivator phosphatase Pho1 are the reaction that most influences the timing, duration, and strength of ERK-PP activity in the MAPK signaling cascade. The second most influential reaction turns out to be the binding and unbinding of Raf* with MEK. The timing and duration are also influenced by the dephosphorylation of the phosphorylated version MEK-P of MEK by the phosphatase Pho2, whereas the strength is also influenced by the binding and unbinding of Raf* with MEK-P. On the other hand, our SOSA sensitivity results summarized in Table III indicate that the phosphatase Pho1, associated with Raf inactivation, and the kinase MEK are two very important molecular species that are responsible for influencing the timing, duration, and strength of ERK-PP activity in MAPK signaling. Interestingly, the Food and Drug Administration (FDA) has recently approved the use of Sorafenib for the treatment of kidney and liver cancer. This drug is a small molecular inhibitor that targets Raf (as well as other kinases) and induces antiproliferative and proapoptotic effects by influencing ERK activity. ${ }^{53}$ The importance of MEK in regulating the response of the MAPK signaling cascade has been investigated by Mansour et al., ${ }^{54}$ who reported that expression of genetically mutated constitutively active MEK is sufficient to cause cellular transformation. In addition to Pho1 and MEK, our SOSA results reveal two other influential species, namely, MEK-P-Pho2 and MEK-P, which influence the timing/duration and the strength of ERK-PP activity, respectively. Both are key reactant species in the MEK dephosphorylation step of the MAPK cascade that leads to MEK inactivation. It has been reported by Orth et $a{ }^{55}$ that prevention of MEK activation may contribute to eventual apoptosis.

A number of investigators recently studied the sensitivity properties of the MAPK signaling cascade by using derivative-based approaches. In particular, Mayawala et al. ${ }^{56}$ considered three response characteristics associated with ERK-PP activity, namely, decay time, peak time, and amplitude gain. We can relate these characteristics to the duration, timing, and strength of ERK-PP activity, respectively. Mayawala et al. concluded that the decay time (duration) is most sensitive to phosphatase reactions at the MEK level and that these reactions do not significantly influence the amplitude 
ROSOSA

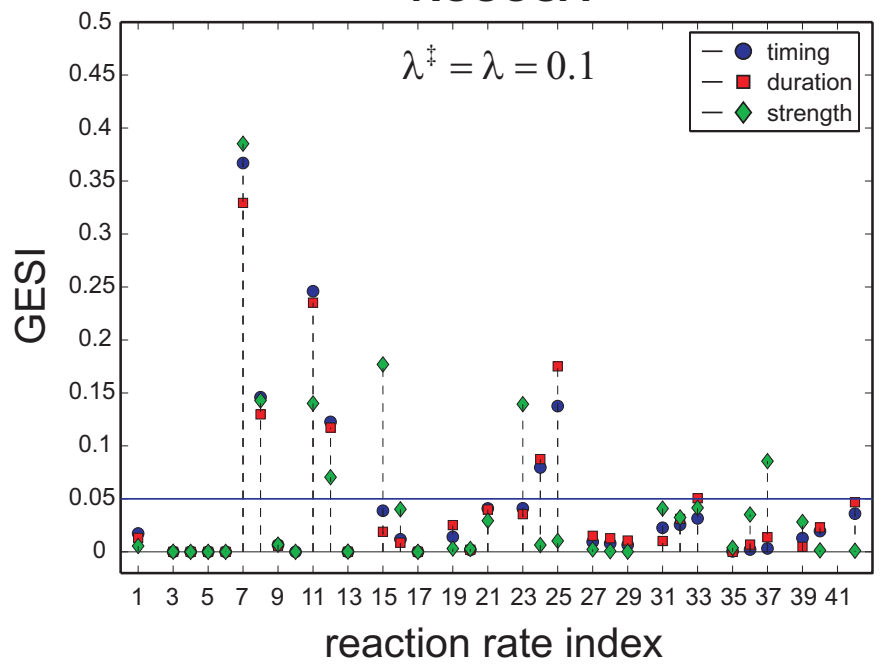

ROSOSA

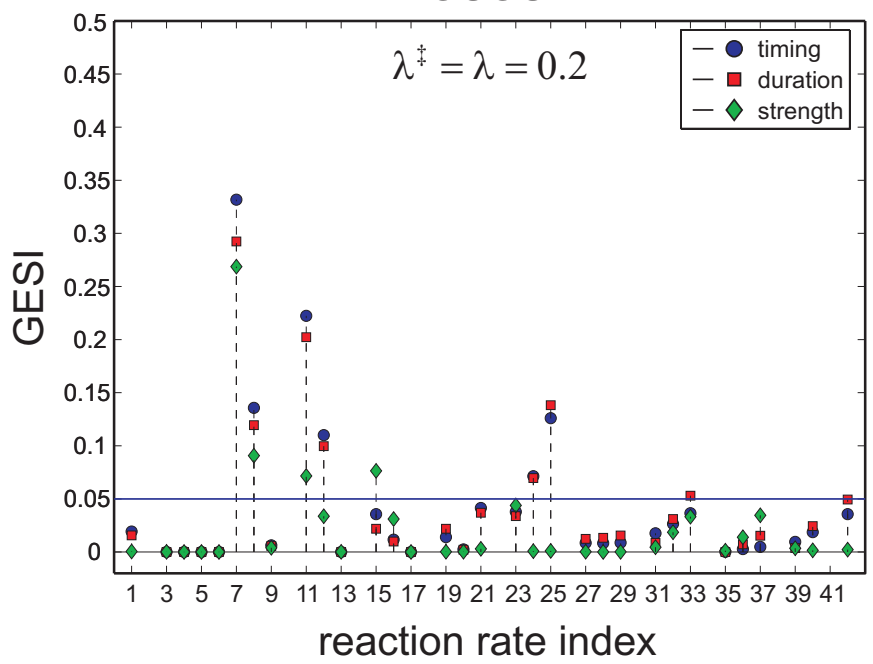

ROSOSA

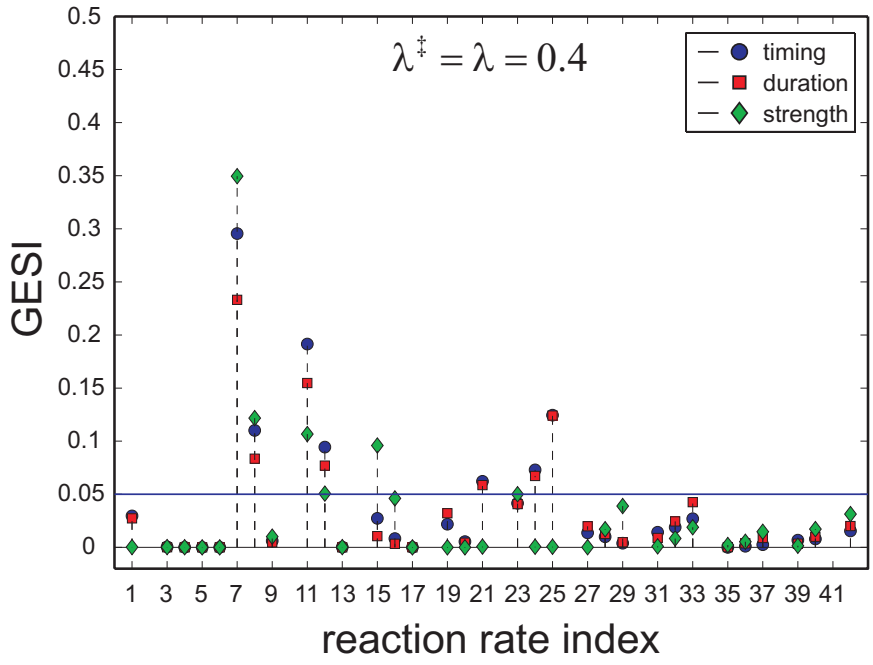

FIG. 6. ROSOSA reaction rate results for the MAPK cascade and for three different fluctuation levels with $\lambda^{\ddagger}=\lambda=0.1,0.2,0.4$. The GESI values associated with the reaction rate constants $\kappa_{2}, \kappa_{10}, \kappa_{14}, \kappa_{18}, \kappa_{22}, \kappa_{26}, \kappa_{30}, \kappa_{34}, \kappa_{38}$, and $\kappa_{41}$ are not calculated, since these constants are zero (they correspond to the reverse portion of the irreversible reactions $1,5,7,9,11,13,15,17,19$, and 21).

gain (strength). These conclusions are in agreement with our results obtained by variance-based sensitivity analysis. Mayawala et al. also concluded that the amplitude gain (strength) is most sensitive to phosphatase reactions at the ERK level, whereas there is no reaction that influences the peak time (timing). Unfortunately, these conclusions are not supported by our study. Recall that for small fluctuations (i.e., when $\lambda^{\ddagger}=0.1,0.2$ ), the ROSA results indicate that reaction 19

TABLE IV. MAPK ROSOSA results.

\begin{tabular}{|c|c|c|c|c|}
\hline Rate & Reaction & $T$ & $D$ & $S$ \\
\hline$\kappa_{7}$ & $\mathrm{Raf}^{*}+$ Pho1 $\rightarrow$ Raf $^{*}-$ Pho1 & $\bullet$ & $\bullet$ & $\bullet$ \\
\hline$\kappa_{8}$ & Raf $^{*}-$ Pho $1 \rightarrow$ Raf $^{*}+$ Phol & • & $\bullet$ & $\bullet$ \\
\hline$\kappa_{11}$ & $\mathrm{MEK}+\mathrm{Raf}^{*} \rightarrow \mathrm{MEK}-\mathrm{Raf}^{*}$ & $\bullet$ & $\bullet$ & $\bullet$ \\
\hline$\kappa_{12}$ & $\mathrm{MEK}-\mathrm{Raf}^{*} \rightarrow \mathrm{MEK}+\mathrm{Raf}^{*}$ & $\bullet$ & $\bullet$ & $\bullet$ \\
\hline$\kappa_{15}$ & $\mathrm{MEK}-\mathrm{P}+\mathrm{Raf}^{*} \rightarrow \mathrm{MEK}-\mathrm{P}-\mathrm{Raf}^{*}$ & & & - \\
\hline$\kappa_{23}$ & $\mathrm{MEK}-\mathrm{P}+\mathrm{Pho} 2 \rightarrow \mathrm{MEK}-\mathrm{P}-\mathrm{Pho} 2$ & & & $\bullet$ \\
\hline$\kappa_{24}$ & $\mathrm{MEK}-\mathrm{P}-\mathrm{Pho} 2 \rightarrow \mathrm{MEK}-\mathrm{P}+\mathrm{Pho} 2$ & • & • & \\
\hline$\kappa_{25}$ & $\mathrm{MEK}-\mathrm{P}-\mathrm{Pho} 2 \rightarrow \mathrm{MEK}+\mathrm{Pho} 2$ & $\bullet$ & - & \\
\hline
\end{tabular}

$($ ERK $-\mathrm{PP}-\mathrm{Pho} 3 \rightarrow \mathrm{ERK}-\mathrm{P}+\mathrm{Pho}$ - dephosphorylation of ERK-PP) influences the strength of ERK-PP activity, whereas for large fluctuations (i.e., when $\lambda^{\ddagger}=0.4$ ), reaction 21 (ERK-P-Pho3 $\rightarrow$ ERK + Pho3-dephosphorylation of ERK-P) influences the strength of ERK-PP activity; see Fig. 3. However, our results (data not shown) reveal that the influence of reaction 19 accounts for only $13 \%$ for $\lambda^{\ddagger}=0.1$ and $17 \%$ for $\lambda^{\ddagger}=0.2$ of the output variance, whereas the influence of reaction 21 accounts for only $18 \%$ of the output variance.

On the other hand, Liu et al. ${ }^{16}$ identified the activation/ inactivation of Raf and several reactions associated with the phosphorylation and dephosphorylation of MEK as being very influential in shaping the system output. Their conclusions are in agreement with our results obtained by variancebased sensitivity analysis.

Finally, Hornberg et al. ${ }^{15}$ concluded that most reactions in the MAPK cascade are not important for influencing the output ERK-PP profile. However, they have noted that Raf inactivation and MEK phosphorylation by Raf are the most 
influential processes for controlling the duration, integrated response (related to timing), and amplitude (related to strength) of ERK-PP activity. Hornberg et al. also noted that the amplitude is also controlled by ERK phosphorylation using MEK, whereas the duration and integrated response are further controlled by MEK dephosphorylation. Moreover, they have indicated the importance of MEK, ERK, Pho1, Pho2, and Pho3 for influencing the duration and integrated response, as well as the importance of Pho1 and MEK for influencing the amplitude. Finally, Hornberg et al. noted a high correlation between the control of duration and integrated response and very little correlation between the control of duration and amplitude. In general, these results are in agreement with our results obtained by variance-based sensitivity analysis. However, our results clearly indicate that reaction 4 ( Raf* $^{*}+$ Pho1 $\left.\rightleftarrows \mathrm{Raf}^{*}-\mathrm{Pho} 1\right)$ is the most influential reaction for controlling the timing, duration, and strength of ERK-PP activity.

Differences between the previous results and the sensitivity analysis results presented in this paper are partly due to the fact that various studies use different choices for the response characteristics. We also believe that another source of discrepancy is the use of derivatives for sensitivity analysis by the previously mentioned methods. But the most serious problem seems to be the fact that these methods do not employ a thermodynamically consistent approach to sensitivity analysis. It has been recently pointed out by Ederer and Gilles $^{22}$ that thermodynamic inconsistencies may lead to erroneous sensitivity analysis results, which may in turn lead to misleading biological conclusions. The method presented in this paper effectively addresses this important problem.

The issue of thermodynamic consistency has also been discussed by Liebermeister and $\operatorname{Klipp}^{57}$ as well as by Schaber et al. ${ }^{58}$ who proposed an alternative approach to the one presented in this paper for modeling the reaction rate constants of a biochemical reaction system. Their formulation is based on the well-known Haldane relation of enzyme catalyzed reactions. ${ }^{59}$ However, derivation of the Haldane relation relies on several assumptions and simplifications, which may not be valid in general. For this reason, we chose to work here with the Eyring-Polanyi equations, which are derived by means of the well-known activated-complex theory, which is one of the most commonly used theories of chemical kinetics. ${ }^{30}$

The results obtained by the sensitivity analysis methodology presented in this paper depend on the particular response characteristic used. In general, different response characteristics will lead to different sensitivity analysis results. Therefore, choosing an appropriate response characteristic is a very important issue when using the variance-based sensitivity analysis methodology presented in this paper. The response characteristic should not be chosen arbitrarily but after careful consideration of the biological problem at hand. However, any computable quantity that is thought to be important in influencing cellular function can serve as a useful response characteristic. This could be the entire time-varying concentration profile of a particular molecular species of interest (which leads to a time-dependent sensitivity analysis approach similar to the one suggested by Leloup and
Goldbeter ${ }^{20}$ ), numerical features extracted from the profile, such as steady-state concentration or timing, duration, and strength (as we did in this paper), or even numerical characteristics extracted from the flux of a selected reaction.

We should finally point out that experimental evidence may suggest that a particular cellular behavior of interest is controlled by the combined influence of the concentration profiles of two (or more) molecular species (e.g., Raf* and ERK-PP in the MAPK cascade). A simple way to deal with this case is to separately calculate the SESIs $\sigma_{\dot{j}}^{(1)}$ and $\sigma_{j}^{(2)}$, the TESIs $\tau_{j}^{(1)}$ and $\tau_{j}^{(2)}$, and the JESIs $\eta_{j}^{(1)}$ and $\eta_{j}^{(2)}$, corresponding to the concentration profiles of two molecular species, say, 1 and 2 , and set $\sigma_{j}=\max \left\{\sigma_{j}^{(1)}, \sigma_{j}^{(2)}\right\}$ and $\eta_{j}$ $=\max \left\{\eta_{j}^{(1)}, \eta_{j}^{(2)}\right\}$. It is not difficult to see that the rules for interpreting the variance-based sensitivity indices summarized in Table I are applied here as well. For example, if $\eta_{j}$ $\simeq 0$ and $\sigma_{j} \simeq 0$, then we may say that factor $j$ does not appreciably influence system behavior. On the other hand, if $\eta_{j} \simeq 0$ and $\sigma_{j} \neq 0$, we may say that factor $j$ influences system behavior mostly singularly. In this case, however, we can get additional information about the actual source of influence by considering the individual SESI values. For example, if $\eta_{j} \simeq 0$ and $\sigma_{j} \neq 0$, with $\sigma_{j}^{(1)} \neq 0$ and $\sigma_{j}^{(2)} \simeq 0$, then we can say that factor $j$ influences the system behavior mostly singularly through the activity of only the first molecular species, whereas if $\sigma_{j}^{(1)} \neq 0$ and $\sigma_{j}^{(2)} \neq 0$, then we can say that factor $j$ influences the system behavior mostly singularly through the activity of both molecular species.

\section{CONCLUSION}

In this paper, we presented a thermodynamically consistent variance-based approach for sensitivity analysis of biochemical reaction systems. Our results clearly show that the proposed method is very appealing, since it can produce rich information about the sensitivity properties of a biochemical reaction system and can lead to rigorous and systematic interpretation of the results. However, variance-based sensitivity analysis is computationally intensive due to the need for a large number of system simulations in a Monte Carlo framework. This problem becomes very serious for large biochemical reaction systems. As a matter of fact, the Monte Carlo estimation approach discussed in Sec. $\mathrm{V}$ requires $2 L(J+1)$ system evaluations [by solving the system of $N$ ordinary differential equations given by Eq. (6)], where $L$ is the number of Latin hypercube samples used and $J$ is the number of biochemical factors considered $(J=M$ for ROSA, $J=N$ for SOSA, and $J=M+N$ for ROSOSA). As a consequence, the simulations presented in Sec. VI required 132000 system evaluations for ROSA, 144000 system evaluations for SOSA, and 270000 system evaluations for ROSOSA. We therefore think that a very important issue for future research is the development of a fast approach to variance-based sensitivity analysis that can be used to efficiently implement the method discussed in this paper. ${ }^{60}$ 


\section{ACKNOWLEDGMENTS}

The authors acknowledge the National Science Foundation (NSF) for support of this research. Special thanks to Garrett Jenkinson for fruitful discussions and suggestions.

${ }^{1}$ H. Kitano, Science 295, 1662 (2002).

${ }^{2}$ H. Kitano, Nature (London) 420, 206 (2002).

${ }^{3}$ I. Nestorov, Clin. Pharmacokinet. 42, 883 (2003).

${ }^{4}$ A. Varma, M. Morbidelli, and H. Wu, Parametric Sensitivity in Chemical Systems (Cambridge University Press, Cambridge, 1999).

${ }^{5}$ A. Saltelli, M. Ratto, S. Tarantola, and F. Campolongo, Chem. Rev. (Washington, D.C.) 105, 2811 (2005).

${ }^{6}$ H. Xu and S. Rahman, Probab. Eng. Mech. 20, 239 (2005).

${ }^{7}$ H. Liu, W. Chen, and A. Sudjianto, J. Mech. Des. 128, 326 (2006).

${ }^{8}$ A. Saltelli and S. Tarantola, J. Am. Stat. Assoc. 97, 702 (2002).

${ }^{9}$ A. Fassò, E. Esposito, E. Porcu, A. P. Reverberi, and F. Vegliò, Environmetrics 14, 743 (2003).

${ }^{10}$ D. Krewski, Y. Wang, S. Bartlett, and K. Krishnan, J. Biopharm. Stat. 5, 235 (1995).

${ }^{11}$ H. Rabitz, M. Kramer, and D. Dacol, Annu. Rev. Phys. Chem. 34, 419 (1983).

${ }^{12}$ J. Zádor, I. G. Zsély, and T. Turányi, Reliab. Eng. Syst. Saf. 91, 1232 (2006).

${ }^{13}$ E. E. Leamer, Modelling Economic Series: Readings in Econometric Methodology (Oxford University Press, New York, 1991), pp. 29-49.

${ }^{14}$ E. E. Leamer, Modelling Economic Series: Readings in Econometric Methodology (Oxford University Press, New York, 1991), pp. 88-96.

${ }^{15}$ J. J. Hornberg, B. Binder, F. J. Bruggeman, B. Schoeberl, R. Heinrich, and H. V. Westerhoff, Oncogene 24, 5533 (2005).

${ }^{16}$ G. Liu, M. T. Swihart, and S. Neelamegham, Bioinformatics 21, 1194 (2005)

${ }^{17}$ D. Hu and J.-M. Yuan, J. Phys. Chem. A 110, 5361 (2006).

${ }^{18}$ A. Mahdavi, R. E. Davey, P. Bhola, T. Yin, and P. W. Zandstra, PLOS Comput. Biol. 3, e130 (2007).

${ }^{19}$ X.-J. Feng, S. Hooshangi, D. Chen, G. Li, R. Weiss, and H. Rabitz, Biophys. J. 87, 2195 (2004).

${ }^{20}$ J.-C. Leloup and A. Goldbeter, J. Theor. Biol. 230, 541 (2004).

${ }^{21}$ H. V. Westerhoff, Systems Biology: Applications and Perspectives (Springer, New York, 2007), pp. 45-67.

${ }^{22}$ M. Ederer and E. D. Gilles, Biophys. J. 92, 1846 (2007).

${ }^{23}$ A. Saltelli, M. Ratto, T. Andres, F. Campolongo, J. Cariboni, D. Gatelli, M. Saisana, and S. Tarantola, Global Sensitivity Analysis: The Primer (Wiley, New York, 2008).

${ }^{24}$ I. M. Sobol', Mathematical Modeling and Computational Experiment 1, 407 (1993).

${ }^{25}$ I. M. Sobol', Math. Comput. Simul. 55, 271 (2001).

${ }^{26}$ A. Saltelli, S. Tarantola, F. Campolongo, and M. Ratto, Sensitivity Analysis in Practice: A Guide to Assessing Scientific Models (Wiley, New York, 2004).

${ }^{27}$ R. Heinrich and S. Schuster, The Regulation of Cellular Systems (Chapman and Hall, London, 1996).

${ }^{28}$ If the concentration $x_{n}(t)$ does not converge to zero within the time interval $\left[0, t_{\max }\right]$, then we set $t_{0}=t_{\max }$.

${ }^{29}$ See EPAPS supplementary material at http://dx.doi.org/10.1063/ 1.3205092 for details.

${ }^{30}$ R. S. Berry, S. A. Rice, and J. Ross, Physical Chemistry, 2nd ed. (Oxford University Press, New York, 2000).

${ }^{31}$ The standard chemical potential defines a reference level with respect to which changes in the chemical potential are measured.

${ }^{32}$ In this paper, we use capital letters to denote random variables and small letters to denote their realizations (samples).
${ }^{33}$ X.-S. Xie and H.-P. Lu, J. Biol. Chem. 274, 15967 (1999).

${ }^{34}$ X.-S. Xie, J. Chem. Phys. 117, 11024 (2002).

${ }^{35}$ J. M. Berg, J. L. Tymoczko, and L. Stryer, Biochemistry, 6th ed. (Freeman, San Francisco, 2006).

${ }^{36}$ Implicit in Eq. (14) is the assumption that, once the values of the random variables $\left\{Y_{m}^{\ddagger}, m=1,2, \ldots, M\right\}$ and $\left\{Y_{n}, n=1,2, \ldots, N\right\}$ are determined, these values remain constant during a time period of interest. It has been argued [M. O. Vlad, G. Cerofolini, P. Oefner, and J. Ross, J. Phys. Chem. B 109, 21241 (2005)] that this is reasonable if we assume that the biochemical reaction system exhibits only static (and not dynamic) disorder.

${ }^{37}$ T. Grönholm and A. Annila, Math. Biosci. 210, 659 (2007).

${ }^{38}$ J. C. Helton and F. J. Davis, Reliab. Eng. Syst. Saf. 81, 23 (2003).

${ }^{39}$ A multivariate function is said to be additive if it can be decomposed into a sum of one-dimensional functions of one variable.

${ }^{40}$ T. Homma and A. Saltelli, Reliab. Eng. Syst. Saf. 52, 1 (1996)

${ }^{41}$ A. Saltelli, S. Tarantola, and F. Campolongo, Stat. Sci. 15, 377 (2000).

${ }^{42}$ R. L. Iman, Risk Anal. 7, 21 (1987).

${ }^{43}$ We use the notation $x \neq 0$ to denote that $x$ may take values that are sufficiently larger than zero.

${ }^{44}$ M. Stein, Technometrics 29, 143 (1987).

${ }^{45}$ M. D. McKay, W. J. Conover, and R. J. Beckman, Technometrics 42, 55 (2000).

${ }^{46}$ J. C. Helton, J. D. Johnson, C. J. Sallaberry, and C. B. Storlie, Reliab. Eng. Syst. Saf. 91, 1175 (2006).

${ }^{47}$ To draw a Latin hypercube sample $\left\{w_{k}^{(q)}, k=1,2, \ldots, K\right\}, q=1,2, \ldots, Q$ of $K$ independent random variables $W_{k}, k=1,2, \ldots, K$ of size $Q$, we first draw $K Q$ samples $\left\{\xi_{k}^{(q)}, k=1,2, \ldots, K, q=1,2, \ldots, Q\right\}$ independently from the uniform distribution on $[0,1]$ and then set $w_{k}^{(q)}=F_{k}^{-1}\left[\left(\pi_{k}^{(q)}-1\right.\right.$ $\left.\left.+\xi_{k}^{(q)}\right) / Q\right]$, where $F_{k}[\cdot]$ is the cumulative distribution function of random variable $W_{k}$ and $\left\{\pi_{k}^{(q)}, q=1,2, \ldots, Q\right\}$ is an independent random permutation of $\{1,2, \ldots, Q\}$.

${ }^{48}$ A. Saltelli, Comput. Phys. Commun. 145, 280 (2002).

${ }^{49}$ L. Chang and M. Karin, Nature (London) 410, 37 (2001).

${ }^{50}$ B. Schoeberl, C. Eichler-Jonsson, E. D. Gilles, and G. Müller, Nat. Biotechnol. 20, 370 (2002).

${ }^{51}$ Equation (14) implies that an increase in the chemical potential value of the activated complex of a reaction one standard deviation from the (zero) mean produces a variation in the reaction rate constant that amounts to $100 e^{\lambda^{\ddagger}} \%$ of the nominal value. As a consequence, the values of $\lambda^{\ddagger}$ considered in this paper correspond to perturbing the reaction rate constants more than $10 \%, 20 \%$, and $50 \%$ of their nominal values when

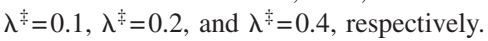

${ }^{52}$ In this case, the TESI is approximately equal to the SESI and, therefore, the JESI is approximately zero. Note also that Eq. (34) implies that the SESI is independent of the standard deviations $\lambda_{j}$ when their values are all equal.

${ }^{53}$ S. M. Wilhelm, L. Adnane, P. Newell, A. Villanueva, J. M. Llovet, and M. Lynch, Molecular Cancer Therapeutics 7, 3129 (2008).

${ }^{54}$ S. J. Mansour, W. T. Matten, A. S. Hermann, J. M. Candia, S. Rong, K. Fukasawa, G. F. Vande Woude, and N. G. Ahn, Science 265, 966 (1994).

${ }^{55}$ K. Orth, L. E. Palmer, Z. Q. Bao, S. Stewart, A. E. Rudolph, J. B. Bliska, and J. E. Dixon, Science 285, 1920 (1999).

${ }^{56}$ K. Mayawala, C. A. Gelmi, and J. S. Edwards, Biophys. J. 87, L01 (2004).

${ }^{57}$ W. Liebermeister and E. Klipp, IEE Proc: Sys. Biol. 152, 97 (2005).

${ }^{58}$ J. Schaber, W. Liebermeister, and E. Klipp, IET Sys. Biol. 3, 1 (2009).

${ }^{59}$ A. Illanes, C. Altamirano, and L. Wilson, Enzyme Biocatalysis: Principles and Applications (Springer, New York, 2008), pp. 107-154.

${ }^{60}$ The sensitivity analysis techniques discussed in this paper have been coded in MATLAB. Interested readers can request a copy of the software and the entire set of data obtained with this software by contacting the corresponding author at goutsias@jhu.edu. 